\title{
Comparison of a coupled near- and far-wake model with a free-wake vortex code
}

\author{
Georg Pirrung ${ }^{1}$, Vasilis Riziotis ${ }^{2}$, Helge Madsen ${ }^{1}$, Morten Hansen ${ }^{1}$, and Taeseong Kim ${ }^{1}$ \\ ${ }^{1}$ Wind Energy Department, Technical University of Denmark, Frederiksborgvej 399, 4000 Roskilde, Denmark \\ ${ }^{2}$ School of Mechanical Engineering, National Technical University of Athens, 9 Heroon Polytechneiou Str.,
} 15780, Athens, Greece

Correspondence to: Georg Pirrung (gepir@dtu.dk)

Received: 12 February 2016 - Published in Wind Energ. Sci. Discuss.: 10 May 2016

Accepted: 16 September 2016 - Published: 20 January 2017

\begin{abstract}
This paper presents the integration of a near-wake model for trailing vorticity, which is based on a prescribed-wake lifting-line model proposed by Beddoes (1987), with a blade element momentum (BEM)based far-wake model and a 2-D shed vorticity model. The resulting coupled aerodynamics model is validated against lifting-surface computations performed using a free-wake panel code. The focus of the description of the aerodynamics model is on the numerical stability, the computation speed and the accuracy of unsteady simulations. To stabilize the near-wake model, it has to be iterated to convergence, using a relaxation factor that has to be updated during the computation. Further, the effect of simplifying the exponential function approximation of the near-wake model to increase the computation speed is investigated in this work. A modification of the dynamic inflow weighting factors of the far-wake model is presented that ensures good induction modeling at slow timescales. Finally, the unsteady airfoil aerodynamics model is extended to provide the unsteady bound circulation for the near-wake model and to improve the modeling of the unsteady behavior of cambered airfoils. The model comparison with results from a free-wake panel code and a BEM model is centered around the NREL $5 \mathrm{MW}$ reference turbine. The response to pitch steps at different pitching speeds is compared. By means of prescribed vibration cases, the effect of the aerodynamic model on the predictions of the aerodynamic work is investigated. The validation shows that a BEM model can be improved by adding near-wake trailed vorticity computation. For all prescribed vibration cases with high aerodynamic damping, results similar to those obtained by the free-wake model can be achieved in a small fraction of computation time with the proposed model. In the cases with low aerodynamic damping, the addition of trailed vorticity modeling shifts the results closer to those obtained by using the free-wake code, but differences remain.
\end{abstract}

\section{Introduction}

This work is based on a coupled aerodynamics model, where the trailed vorticity effects in the near wake are computed based on a model proposed by Beddoes (1987), and the farwake contribution is computed using the well-known blade element momentum (BEM) theory. The near-wake model (NWM) is a simplified prescribed-wake lifting-line model which efficiently computes the induction due to the vorticity trailed during a quarter of a rotor revolution. The coupled model can be seen as a hybrid code between a traditional BEM model and the more complex vortex codes. Because a BEM model is based on an actuator disk assumption, it can not model the detailed dynamic induction response at the individual blades. Therefore, the NWM is introduced to model these unsteady induction characteristics due to load changes by pitch, eigenmotion of the blades, turbulent inflow and shear. The accuracy of the computations is improved due to the added aerodynamic coupling between airfoil sections through the trailed vorticity, alleviating the limitations of the BEM strip theory. Especially in cases with large radial load gradients, for example close to trailing edge flaps or other aerodynamic devices or close to the blade root and 
tip, the cross-sectional coupling will lead to an improved prediction of the steady and dynamic induction. The addition of the near-wake model in an aeroservoelastic code has an acceptable effect on the total computation speed. An aeroelastic simulation with the wind turbine code HAWC2 (Larsen and Hansen, 2007; Larsen et al., 2013; Kim et al., 2013), of the DTU 10 MW turbine (Bak et al., 2012) in normal operation with turbulent inflow, takes roughly $10 \%$ (30 aerodynamic sections) to $40 \%$ (55 aerodynamic sections) longer if the near-wake model is enabled than if a pure BEM model is used.

The coupled model using the modified BEM approach for the far wake has been proposed by Madsen and Rasmussen (2004) and extended by Andersen (2010). Further, improvement has been presented by Pirrung et al. (2012), where an iterative procedure was used to ensure convergence and avoid numerical instabilities of the NWM. An application of the coupled model to estimate the critical flutter speeds of the NREL 5 MW turbine (Jonkman et al., 2009), also including blades with modified stiffness, has been described by Pirrung et al. (2014), where the coupled aerodynamics model has predicted $4-10 \%$ higher critical flutter speeds than the unsteady BEM model in the aeroservoelastic wind turbine code HAWC2.

In the present paper, the iteration procedure of the NWM used by Pirrung et al. (2012) is presented in more detail, as well as a method to compute the necessary relaxation factor during a simulation. The presented approach removes the need for additional input or very conservative relaxation factors that are independent of spatial and temporal discretization and increase the computation time. Further, the NWM is simplified to accelerate the computations with small loss of accuracy of the unsteady results.

The dynamic responses to pitch steps and prescribed blade vibrations are validated by comparing them to results from the more complex free-wake code GENUVP (Voutsinas, 2006). The focus in the pitch step cases is the dynamic induction response, while the prescribed vibration cases are evaluated based on aerodynamic work during a period of oscillation. It is found that the coupled aerodynamic model is capable of producing results that agree much better with results obtained from the free-wake code than the unsteady BEM model in most cases, without a dramatic increase in computation time. The more accurate computation of aerodynamic work can have a considerable impact on the aeroelastic response in the case where the total damping is close to zero, such as for edgewise vibrations.

This paper is structured as follows: in the next section a short description of the NWM and a previous implementation of the coupling to a far-wake model and shed vorticity model are presented. An overview of the current implementation is given in Sect. 3. In Sect. 4, modifications to far wake and shed vorticity model are proposed to improve the interaction of these models with the near-wake model and to increase the accuracy of the dynamic lift computation for cambered airfoils. This is followed by a description of the iterative procedure to stabilize the near-wake model in Sect. 5 . A way of simplifying the NWM to accelerate the computation is presented in Sect. 6. In Sect. 7 the free-wake panel code used for validation of the coupled near- and far-wake model is briefly described. The effects of the model modifications and results from the code comparison are shown and discussed in Sect. 8. An overview of the symbols used in the equations and figures is included as Appendix A.

\section{Original model description}

The structure of the previous implementation (Madsen and Rasmussen, 2004; Andersen, 2010) of the model is shown in Fig. 1. From the velocity triangle, denoted as VT, follows a geometric angle of attack (AOA) $\alpha_{\mathrm{QS}}$ and a relative velocity $v_{\mathrm{r}}$. An effective AOA $\alpha_{\text {eff }}$ is obtained through a 2-D modeling of the shed vorticity effects, which is briefly described in Sect. 2.3. This effective AOA is used to determine the aerodynamic forces and the thrust coefficient $C_{\mathrm{T}}$ and torque coefficient $C_{\mathrm{Q}}$. These coefficients lead to a far-wake induction factor $\boldsymbol{a}_{\mathrm{FW}}$, requiring a coupling factor $k_{\mathrm{FW}}$ as input. Section 2.2 contains the dynamic inflow model, using the weighting factors $A_{1}$ and $A_{2}$, which is used to determine the unsteady far-wake induction $\boldsymbol{u}_{\mathrm{FW}, \mathrm{dyn}}$.

Using this far-wake induction, and the near-wake induction from the previous time step, a new intermediate velocity triangle $\mathrm{VT}_{\mathrm{i}}$ is determined, with a new quasi-steady AOA and relative velocity. These lead to the bound circulation $\Gamma_{\mathrm{QS}}$. The difference in $\Gamma_{\mathrm{QS}}$ between adjacent blade sections, denoted as $\Delta \Gamma$ in the following, determines the trailed vorticity. In the next section it is shown how the induced velocity $\boldsymbol{W}$ due to the near wake, which is added to $\boldsymbol{u}_{\mathrm{FW}}$ to obtain the total induced velocity $\boldsymbol{u}_{\text {tot }}$ at each blade section, follows from the trailed vortices. The total induced velocity will then, in addition to the relative velocity due to blade motion and turbulence in the incoming wind, determine the velocity triangle after the time step $\Delta t$.

\subsection{Near-wake model}

The NWM enables a fast computation of the induction due to the trailed vorticity behind a rotor blade. The trailed wake can be discretized into trailed vortex arcs from several positions on the blade, where each arc consists of a number of vortex elements. The induction at a blade section due to each vortex element can be computed using the Biot-Savart law, but this computation is numerically expensive as the influence of each vortex element on the induction at each blade section has to be determined. Beddoes (1987) proposed to avoid these expensive computations by assuming that the trailed vorticity follows circular vortex arcs in the rotor plane and limiting the computation to a quarter rotation. In this quarter rotation, the axial induction $\mathrm{d} w$ from a vortex element at a blade position is decreasing as the vortex element moves 


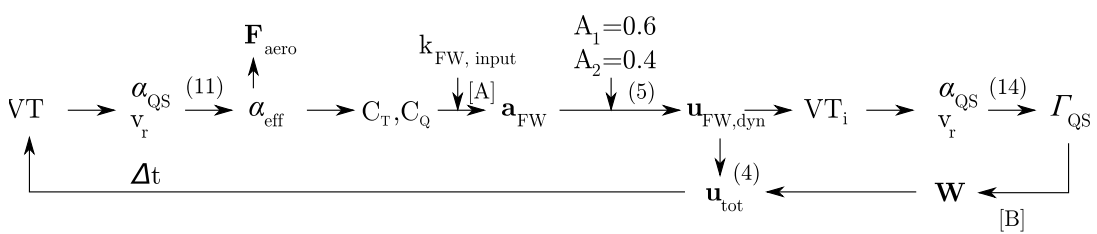

Figure 1. The previous implementation of the coupled near- and far-wake model, as described by Madsen and Rasmussen (2004) and Andersen (2010). The numbers in parentheses refer to the equations in the following sections, [B] to the original model by Beddoes (1987), and $[\mathrm{A}]$ to Andersen (2010). The vector notation on the induction factors and induced velocity indicates that the model can be used to compute both axial and tangential induction.

away from the blade, starting with a value $\mathrm{d} w_{0}$. This decreasing induction, following from the Biot-Savart law, is approximated by exponential functions:

$\frac{\mathrm{d} w}{\mathrm{~d} w_{0}} \approx 1.359 e^{-\beta / \Phi}-0.359 e^{-4 \beta / \Phi}$,

where $\Phi$ is a geometric factor depending on the radius from which the vortex is trailed and the distance between the vortex trailing point and the blade section where the induction is computed. The angle $\beta$ determines how much the blade has rotated away from the vortex element. The numerically efficient trailing wake algorithm gives the axial induction $W$ due to the trailed vorticity at time step $i$ at a blade section $s$ as

$W_{s}^{i}=\sum_{v=1}^{N_{v}} W_{s, v}^{i}$

where $N_{v}$ is the number of vortex arcs trailed from the blade and $W_{s, v}$ is the induction due to a single vortex arc $v$ at the blade section. The induction consists of components $X_{s, v}^{i}$ and $Y_{s, v}^{i}$, corresponding to both of the exponential terms in Eq. (1):

$W_{s, v}^{i}=X_{s, v}^{i}+Y_{s, v}^{i}$,

$X_{s, v}^{i}=X_{s, v}^{i-1} e^{-\Delta \beta_{v} / \Phi_{s, v}}+D_{X, s, v} \Delta \Gamma_{v}\left(1-e^{-\Delta \beta_{v} / \Phi_{s, v}}\right)$,

$Y_{s, v}^{i}=Y_{s, v}^{i-1} e^{-4 \Delta \beta_{v} / \Phi_{s, v}}+D_{Y, s, v} \Delta \Gamma_{v}\left(1-e^{-4 \Delta \beta_{v} / \Phi_{s, v}}\right)$,

where $\Delta \Gamma_{v}$ is the trailed vortex strength, which depends on the radial difference in bound circulation between the blade sections adjacent to the vortex trailing point. The relative movement of the blade in the rotor plane during the time step at the vortex trailing point is denoted as $\Delta \beta_{v}=$ $\left(v_{\mathrm{r}, \text { in-plane }} / r\right) \Delta t$. The in-plane velocity component perpen-

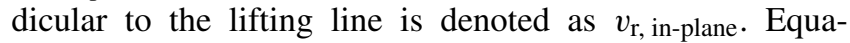
tions ( $3 b)$ and $(3 c)$ show that the induction consists of a decreasing part of the induction at the previous time step, due to the previously trailed wake moving away from the blade, and the contributions from the newest element, given by the $D_{X, s, v}$ and $D_{Y, s, v}$ terms. These equations are less time-stepsensitive and computationally faster than the original equations by Beddoes (1987). They have been proposed by Pirrung et al. (2016), as well as a modification of $\Phi$ to account for the helix angle of the trailed vorticity. Wake expansion could in principle be included by another modification of $\Phi$, but the effect of wake expansion on the near-wake induction of the individual blades is expected to be negligible. Pirrung et al. (2016) also describe how the tangential induction is computed based on the same approach as the axial induction. In Sect. 6 of the present article a reduction of the exponential function approximation, Eq. (1), to a single term is proposed. This simplified and computationally cheaper approach removes the $Y$ component in Eqs. (3a) and (3c).

\subsection{Coupling to far-wake model}

The NWM, which only computes a fraction of the total rotor induction, is complemented by a modified BEM model for the far wake. The total induced velocity at a blade section is computed as

$\boldsymbol{u}_{\mathrm{tot}}=\boldsymbol{u}_{\mathrm{FW}}+\boldsymbol{W}$,

where $\boldsymbol{u}_{\mathrm{FW}}$ is the far-wake component of the induced velocity and $\boldsymbol{W}$ is the near-wake component (see Eq. 2).

The far-wake component $\boldsymbol{u}_{\mathrm{FW}}$ is computed based on the BEM model implementation in HAWC2 that uses a polynomial to relate the local thrust coefficient with the axial induction factor at each annular element (Madsen et al., 2010). Because a part of the total induction is computed by the nearwake model, the BEM implementation used to determine the far-wake induction factor $\boldsymbol{a}_{\mathrm{FW}}$ has been modified in two ways compared to the original BEM implementation in HAWC2. First, the tip loss correction is removed, because the nearwake model accounts for the increasing induced velocity towards the blade tip (Pirrung et al., 2016). Also, prior to computing the induction factors, the thrust coefficient and torque coefficient are reduced by multiplying by a coupling factor $k_{\mathrm{FW}}<1$ to avoid predicting exaggerated induction levels on the whole rotor disk. The coupling factor is automatically adjusted during the computation. The goal of the adjustment is for the coupled near- and far-wake model to closely match the thrust of a reference BEM model. The reference axial induction factor $a_{\text {ref }}$ is computed as in the regular HAWC2 BEM implementation: including tip loss effects and without reducing the thrust and torque coefficient (Pirrung et al., 2016). 


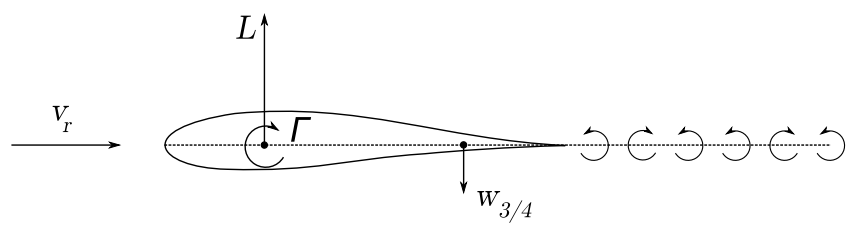

Figure 2. Cambered airfoil in parallel inflow to the chord line. The shed wake corresponds to the time history of the bound circulation.

To account for the far-wake dynamics, this work uses the dynamic inflow model implemented in HAWC2. The model is applied in the same way for axial and tangential induction, so in the following a scalar notation is used for simplicity. Two parallel first-order low-pass filters are applied on the quasi-steady induced velocities $u_{\mathrm{FW}, \mathrm{QS}}=a_{\mathrm{FW}} u_{\infty}$ from the BEM model:

$$
\begin{aligned}
u_{\mathrm{FW}, \mathrm{dyn}}^{i} & =A_{1} u_{1}^{i}+A_{2} u_{2}^{i}, \\
u_{1}^{i} & =u_{1}^{i-1} e^{-\Delta t / \tau_{1}}+u_{\mathrm{FW}, \mathrm{QS}}^{i}\left(1-e^{-\Delta t / \tau_{1}}\right), \\
u_{2}^{i} & =u_{2}^{i-1} e^{-\Delta t / \tau_{2}}+u_{\mathrm{FW}, \mathrm{QS}}^{i}\left(1-e^{-\Delta t / \tau_{2}}\right) .
\end{aligned}
$$

In a pure BEM computation and the previous far-wake model implementation, the factors $A_{i}$ are $A_{1}=0.6$ and $A_{2}=0.4$. They are used to divide the induction into a faster and slower reacting part, corresponding to a faster time constant $\tau_{1}$ and the slower time constant $\tau_{2}$. Both time constants are a function of radius and mean loading. The constants $A_{i}$ and $\tau_{i}$ have been tuned to actuator disk simulations of step changes in uniform loading (Sørensen and Madsen, 2006).

\subsection{Unsteady airfoil aerodynamics model}

The sketch in Fig. 2 illustrates how the shed vorticity due to the time variation in the bound circulation induces a downwash $w_{3 / 4}$ at the three-quarter chord of an airfoil. This downwash will change the angle of attack and thus the lift, drag and moment coefficients according to the airfoil polars, as well as the directions of the aerodynamic forces. The inviscid part of the unsteady airfoil aerodynamics model by Hansen et al. (2004) treats the shed vorticity effects as a time lag on the angle of attack according to Jones' function for a flat plate. The effective angle of attack $\alpha_{\text {eff }}$, which determines the magnitude and direction of the unsteady aerodynamic forces, is computed as

$$
\begin{aligned}
T_{0}^{i} & =\frac{c}{2 v_{\mathrm{r}}^{i}}, \\
x_{1}^{i} & =x_{1}^{i-1} e^{-0.0455 \frac{\Delta t}{T_{0}^{i}}} \\
& +\frac{1}{2}\left(\alpha_{\mathrm{QS}}^{i}+\alpha_{\mathrm{QS}}^{i-1}\right) 0.165 v_{\mathrm{r}}^{i}\left(1-e^{-0.0455 \frac{\Delta t}{T_{0}^{i}}}\right), \\
x_{2}^{i} & =x_{2}^{i-1} e^{-0.3 \frac{\Delta t}{T_{0}^{i}}}
\end{aligned}
$$

$$
\begin{aligned}
& +\frac{1}{2}\left(\alpha_{\mathrm{QS}}^{i}+\alpha_{\mathrm{QS}}^{i-1}\right) 0.335 v_{\mathrm{r}}^{i}\left(1-e^{-0.3 \frac{\Delta t}{T_{0}^{i}}}\right), \\
\alpha_{\mathrm{eff}}^{i} & =\frac{1}{2} \alpha_{\mathrm{QS}}^{i}+\left(x_{1}^{i}+x_{2}^{i}\right) / v_{\mathrm{r}}^{i},
\end{aligned}
$$

where the superscript $i$ denotes the time step and $c$ the chord length. Further, $\alpha_{\mathrm{QS}}$ is the quasi-steady angle of attack resulting from the velocity triangle at the blade section and $v_{\mathrm{r}}$ denotes the corresponding relative velocity.

\section{Model overview}

The structure of the current implementation of the coupled near- and far-wake model is shown in Fig. 3. The changes to the previous implementation (see Fig. 1) are

- The weighting factors $A_{i}$ of the far-wake dynamic inflow are adjusted during the computation to account for the induction computed by the near-wake model, which is explained in Sect. 4.1.

- The trailed vorticity is no longer based on the quasisteady bound circulation $\Gamma_{\mathrm{QS}}$ but instead on a dynamic bound circulation $\Gamma_{\mathrm{dyn}}$. The computation of the dynamic bound circulation is shown in Sect. 4.2.1.

- The near-wake induction is computed in an iteration loop, which is detailed in Sect. 5.

- The coupling factor is no longer needed as input but instead continually updated during the computation, as described by Pirrung et al. (2016).

- The trailed vorticity is assumed to follow helix arcs to account for the downwind convection of the trailed vorticity. To achieve this, $\Phi$ is multiplied by a correction function $f$, depending on the blade section and vortex trailing point, as well as the helix angle at which the vortex is trailed (Pirrung et al., 2016).

- The computation of $\alpha_{\text {eff }}$ according to shed vorticity effects is improved for cambered airfoils, which is explained in Sect. 4.2.2.

\section{Modifications to far-wake and shed vorticity model}

\subsection{Adapting the weighting of the dynamic inflow time filters}

The dynamic inflow model described in Sect. 2.2, which has been tuned for BEM computations, has to be modified if a part of the induction is covered by the NWM. The objective is to obtain a similar slow induction response with the coupled near- and far-wake model as with an unsteady BEM model.

This requires a modification of the constants $A_{1}$ and $A_{2}$ in Eq. (5). The new constants $A_{i}$ are computed based on the 


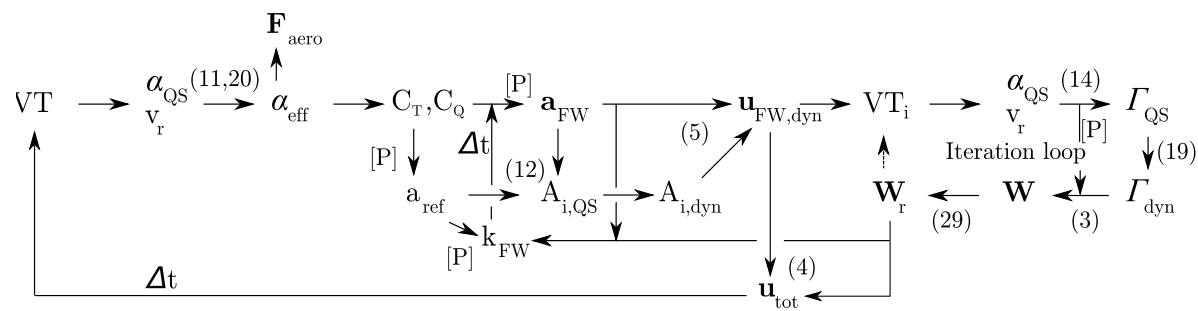

Figure 3. Overview of one time step in the coupled near- and far-wake model used in this work. Relevant equation numbers are included. [P] refers to Pirrung et al. (2016).

far-wake axial induction factor $a_{\mathrm{FW}}$ and a reference axial induction factor obtained from a BEM model with tip loss correction (see Sect. 2.2). The weighting constants for the farwake model are determined such that roughly $40 \%$ of the total axial induction is considered to be reacting slowly, as in the original dynamic inflow model for BEM computation, Eq. (5):

$A_{1, \mathrm{FW}}=\frac{0.4 a_{\mathrm{ref}}}{a_{\mathrm{FW}}}$,

$A_{2, \mathrm{FW}}=1-A_{1, \mathrm{FW}}$.

The factors are continuously updated during the computations. A first-order low-pass filter with the far-wake time constant $\tau_{2}$ of the dynamic inflow model is applied on $A_{1, \mathrm{FW}}$ to make sure this model does not introduce unphysical rapid induction variations due to instantaneous changes in the weighting factors.

\subsection{Extensions of the unsteady airfoil aerodynamics model}

\subsubsection{Unsteady circulation computation}

The influence of shed vorticity on the bound circulation buildup has to be considered when determining the strength of the trailed vortices of the NWM. Joukowski's relation between quasi-steady lift $L_{\mathrm{QS}}$ and circulation $\Gamma_{\mathrm{QS}}$,

$\Gamma_{\mathrm{QS}}=\frac{L_{\mathrm{QS}}}{\rho v_{\mathrm{r}}}=\frac{1}{2} v_{\mathrm{r}} c C_{\mathrm{L}}$,

which has been used by Madsen and Rasmussen (2004) and Andersen (2010) to determine the bound vorticity, is not valid for unsteady conditions. The error of calculating the circulation based on the unsteady lift at an airfoil section depends on the reduced frequency $k=\omega c /\left(2 v_{\mathrm{r}}\right)$, where $\omega$ is the angular velocity, $c$ is the chord length, and $v_{\mathrm{r}}$ is the relative flow speed. For an airfoil pitching harmonically about the threequarter chord point, the error has been estimated by Madsen and Gaunaa (2004) to be $10 \%$ at $k=0.1$ and $100 \%$ at $k=0.8$, which for the NREL $5 \mathrm{MW}$ reference turbine at rated wind and rotor speed corresponds to frequencies of about 1.2 and $9.8 \mathrm{~Hz}$ at $60 \mathrm{~m}$ rotor radius with a chord of $2 \mathrm{~m}$. Except for the first flapwise and edgewise bending frequencies, most relevant modal frequencies for modern blades are between these values, which shows that it is important to include a modeling of the unsteady circulation.

In this paper, the step response of the circulation is approximated by the three-term indicial function used by Madsen and Gaunaa (2004).

$\Gamma_{\mathrm{dyn}} / \Gamma_{\mathrm{QS}}=1-A_{\Gamma, 1} e^{-b_{\Gamma, 1} \tau}-A_{\Gamma, 2} e^{-b_{\Gamma, 2} \tau}-A_{\Gamma, 3} e^{-b_{\Gamma, 3} \tau}$,

where

$\tau=\Delta t \frac{2 v_{\mathrm{r}}}{c}, A_{\Gamma, 1}=0.5547, A_{\Gamma, 2}=0.1828, A_{\Gamma, 3}=0.2656$,

$b_{\Gamma, 1}=0.3064, b_{\Gamma, 2}=0.0439, b_{\Gamma, 3}=3.227$.

In the same way as in the shed vorticity model, the dimensionless time $\tau$ depends on the relative velocity $v_{\mathrm{r}}$, which is updated in each time step. The algorithm is implemented analogue to the computation for the effective angle of attack in Eqs. (8)-(11):

$$
\begin{aligned}
& x_{\Gamma, j}^{i}=x_{\Gamma, j}^{i-1} e^{-b_{\Gamma, j} \frac{\Delta t}{T_{0}^{i}}}+\frac{A_{\Gamma, j}}{2}\left(\Gamma_{\mathrm{QS}}^{i}+\Gamma_{\mathrm{QS}}^{i-1}\right)\left(1-e^{-b_{\Gamma, j} \frac{\Delta t}{T_{0}^{i}}}\right), \\
& \Gamma_{\mathrm{dyn}}^{i}=x_{\Gamma, 1}^{i}+x_{\Gamma, 2}^{i}+x_{\Gamma, 3}^{i},
\end{aligned}
$$

where the quasi-steady circulation is computed from the quasi-steady lift coefficient using Eq. (14).

\subsubsection{Unsteady aerodynamics of cambered airfoils}

Any change in bound circulation $\Gamma$, which is a function of $v_{\mathrm{r}} C_{\mathrm{L}}$ (see Eq. 14) should lead to the corresponding shed vorticity. The implementation of the shed vorticity model according to Hansen et al. (2004) (see Eqs. 9-11) is based on the term $\alpha_{\mathrm{QS}} v_{\mathrm{r}}$. The camber of the airfoil is neglected in this computation of the shed vorticity effects. We propose in this work to replace $\alpha_{\mathrm{QS}}$ in Eqs. (9)-(11) by $\alpha_{\mathrm{QS}}$, camber, with

$\alpha_{\mathrm{QS}, \text { camber }}=\alpha_{\mathrm{QS}}-\alpha_{0}$,

where $\alpha_{0}$ is the zero lift angle of the airfoil.

The impact of this modification is shown for basic cases of relative velocity changes in Figs. 4 and 5, where an airfoil with a $2 \pi$ lift gradient, a $2 \mathrm{~m}$ chord length, a zero lift angle of $-3^{\circ}$ and a drag coefficient of $C_{\mathrm{D}}=0.005$ has been 


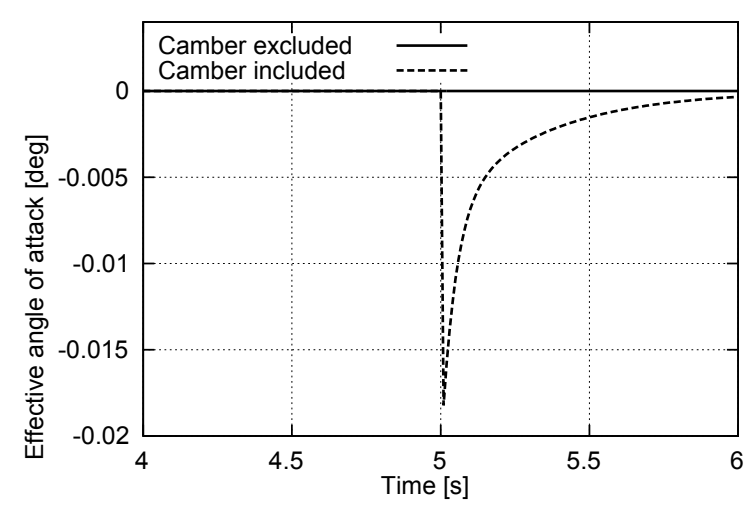

Figure 4. Effect of including camber in the unsteady aerodynamics model on effective angle of attack during a step in relative velocity.

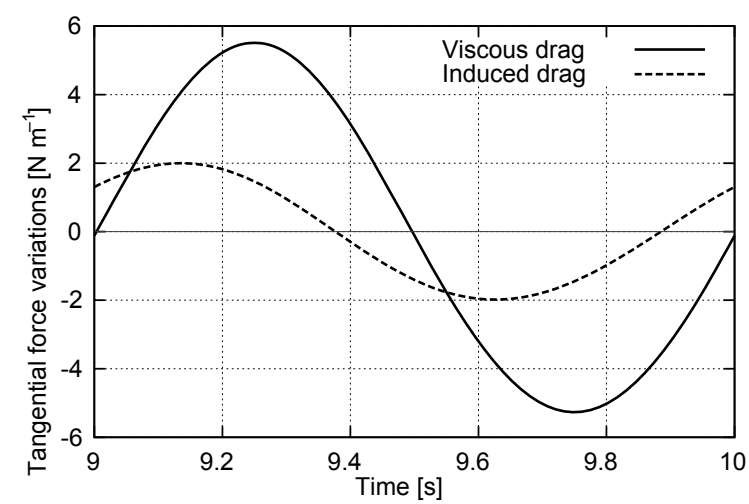

Figure 5. Comparison of viscous drag and induced drag during oscillations of a cambered airfoil parallel to the inflow at $1 \mathrm{~Hz}$. The effect of camber is included as proposed in Eq. (20). The mean drag has been subtracted.

simulated. The airfoil characteristics and chord length have been chosen to be similar to the outboard region of the NREL $5 \mathrm{MW}$ reference turbine, and the geometric angle of attack has been chosen as zero to show the isolated effect of airfoil camber. In Fig. 4, the variation in effective angle of attack due to a step change in relative speed from 70 to $71 \mathrm{~m} \mathrm{~s}^{-1}$ within a time step of $0.01 \mathrm{~s}$ is shown. Without the effect of camber, the change in relative speed has no influence on the angle of attack, because $\alpha_{\mathrm{QS}}$ is a constant zero. The effect of camber leads to a lower angle of attack due to the shed vorticity caused by the increase in bound circulation. The camber effect is small, and the angle of attack changes only by less than $0.02^{\circ}$ immediately after the relative speed step. In Fig. 5 the induced drag due to angle of attack changes is compared to the viscous drag in the case of a vibration of the airfoil section parallel to the inflow. There would be no induced drag in this example if camber was excluded from the effective angle of attack computation. The amplitude of the vibration is $1 \mathrm{~m}$ and the frequency $1 \mathrm{~Hz}$. The effect of induced drag is of the same order of magnitude as the airfoil drag, which indicates the importance of including the airfoil camber in the unsteady airfoil aerodynamics model. The camber effect is included in all further computations presented in this paper except in the left plot of Fig. 18, where it is excluded to investigate its importance for in-plane blade vibrations.

In the unsteady circulation computation described in the previous section, the camber is accounted for through the quasi-steady circulation $\Gamma_{\mathrm{QS}}$, which is based on the lift coefficient (see Eq. 14).

\section{Iterative near-wake and shed vorticity model}

\subsection{Iteration scheme}

The NWM can become numerically unstable depending on the time step, operating point of the turbine, blade geometry and radial calculation point distribution (Pirrung et al., 2012). Figure 7 shows the maximum time step where a stable computation is possible for a fine- and coarse-geometry definition, shown in Fig. 6, of the NREL 5 MW blade. The coarsegeometry definition is a blade geometry typically distributed for BEM computations and the fine distribution is more suitable for computations with higher-fidelity codes. The aerodynamic calculation points and vortex trailing points follow a cosine distribution, which means they are placed at equiangle increments. The time steps have been determined in a numerical experiment, where the time step has been decreased until large oscillations of the induction disappear. The results are accurate to the first significant digit. It can be seen that the finer blade geometry leads to a more stable computation. This can be explained by the smoother blade tip, where the blade chord is approaching zero. Thus, the radial circulation gradient at the very blade tip is smaller and the vortex strength of the tip vortex is distributed to several weaker trailed vortices in the tip region that are less likely to cause numerical instabilities. In a coupled aeroelastic simulation, the small stable time steps for resolutions of 30-60 points would lead to a very slow computation, especially in the case of the coarser blade geometry.

The numerical instability which occurs at larger time steps can be explained as follows: the axial induction due to trailed vortices typically reduces the angle of attack at a blade section, which in attached flow leads to a reduced lift. In the original implementation of the NWM the constant circulation trailed during a time step is only depending on the flow conditions at the blade at the beginning of a time step. Therefore, a longer time step will lead to a larger induction and thus a further reduction in lift in the next time step. If the time step is too large, the induction can become large enough to create a negative lift in the next time step that is larger in absolute value than the previous positive lift. This in turn leads to stronger trailed vortices of opposite sign, which will cause even larger induced velocities in the opposite direction, which again leads to stronger vortices. 


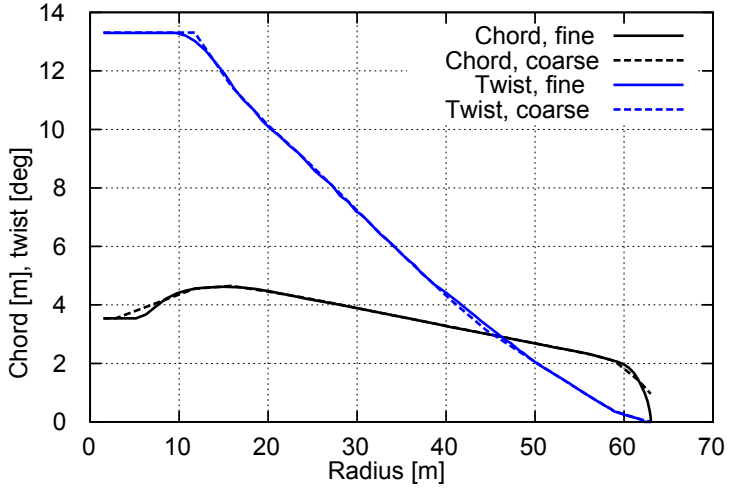

Figure 6. Coarse and fine blade geometry for the NREL $5 \mathrm{MW}$ reference turbine. The coarse definition is a typical geometry definition for BEM-based computations. The finer geometry is smoothed for use in computational fluid dynamics and free-wake codes.

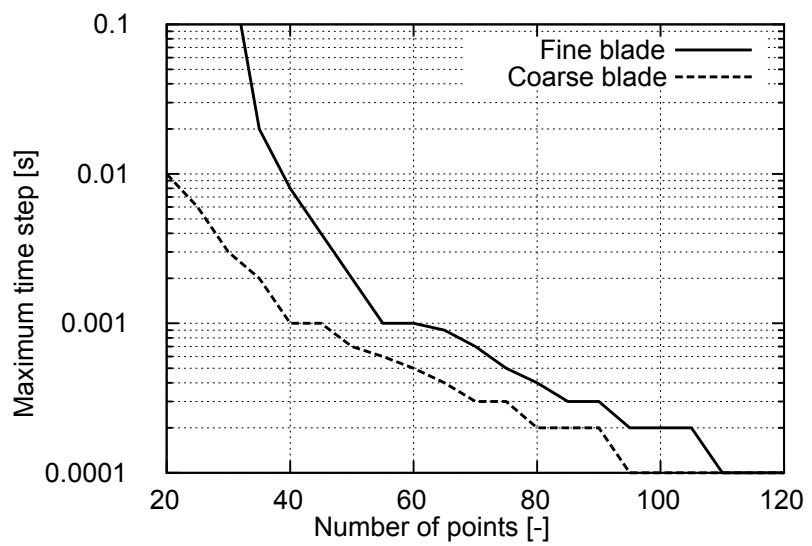

Figure 7. Maximum stable time step depending on the number of points for the coarse and fine blade geometries of the NREL 5 MW reference turbine. The points are distributed using a full cosine distribution (Pirrung et al., 2012). The results are obtained through a numerical experiment.

To stabilize the NWM the balance between trailed vortex strength based on the sectional circulation and the induced velocities are iterated to equilibrium in each time step, which removes the need for small time steps to stabilize the aerodynamics model. The iteration is structured as follows:

1. The quasi-steady circulation is computed according to Joukowski's law using the velocity triangle at the airfoil section based on the induction from the last iteration.

2. The unsteady circulation is computed including shed vorticity effects (see Sect. 4.2).

3. This unsteady circulation defines the constant vortex strengths trailed during a time step.

4. These constant vortex strengths lead to an induction at all airfoil sections.
5. The new induction is combined from the inductions from step 1 and 4 by applying a relaxation factor: $W_{i}=$ $W_{i-1} f_{\mathrm{r}}+W_{i}\left(1-f_{\mathrm{r}}\right)$, where the subscript $i$ indicates the iteration number. If $W_{i}$ is sufficiently close to $W_{i-1}$, it is the desired converged induction.

The BEM model for the far-wake is excluded from this iteration procedure. The AOA and relative velocity used to compute the far-wake induction are the values from the converged iteration in the previous time step. This is accelerating the computation and is feasible because the near-wake effects are on a much faster timescale than the dynamic inflow effects in the BEM model.

\subsection{Estimation of the necessary relaxation factor}

In the following, an estimation of the relaxation factor for a blade section is described. A conservative estimation is based on the least stable case, which is characterized by the following properties:

- One single blade section with one vortex trailing from each side. Adjacent sections would tend to have similar circulations and therefore reduce the vortex strengths and the corresponding induction at the blade section. The trailed vortices on both sides of the section depend only on the bound circulation $\Gamma$ of that section.

- The lift coefficient is linearly dependent on the angle of attack, $C_{\mathrm{L}}=2 \pi \alpha$. A reduced but still positive gradient due to stall would stabilize the model. Note that a $2 \pi$ lift gradient is only assumed in the relaxation factor estimation proposed in this section. In all other parts of the model the lift coefficients and lift gradients according to the airfoil polars are used.

- No prior trailed vorticity is present. It would stabilize the model, because the induction would be determined not only by the momentary circulation at the section but also by the decaying influence of the wake trailed before. If the model converges in the very first time step, with a given induction at the section from the previous iteration, then the iterations will also converge with prior trailed vorticity.

- The helix angle at which the vortices are trailed is assumed to be small. Thus, all the induction due to trailed vorticity is assumed to be axial induction.

With these assumptions, the downwash after a time step $\Delta t$ can be determined by summing up the contributions of the newest element (see the right terms in Eqs. $3 b$ and 3c), for both adjacent vortices:

$$
\begin{aligned}
W_{i} & =\sum_{v=1}^{2}(-1)^{v} \Gamma \\
& \quad\left(D_{X, v}\left(1-e^{-\Delta \beta / \Phi_{v}}\right)+D_{Y, v}\left(1-e^{-4 \Delta \beta / \Phi_{v}}\right)\right),
\end{aligned}
$$




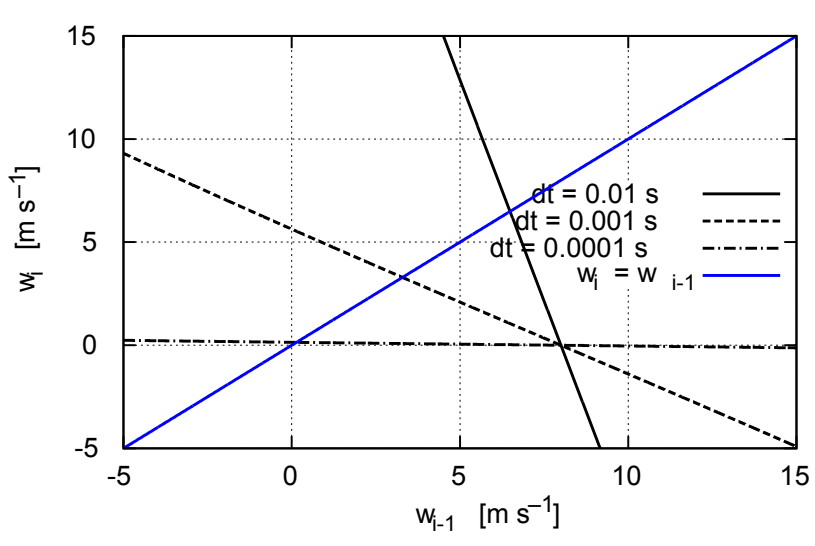

Figure 8. Downwash after an iteration as a function of the downwash from the preceding iteration in the case of a single section with trailed vortices.

where the subscript $v$ denotes the vortex further inboard $(v=1)$ and outboard $(v=2)$ of the section with the bound circulation $\Gamma$. The subscript $i$ denotes the iteration. Because the tangential induction is neglected, $\Delta \beta$ is only a function of the rotation speed of the turbine and the time step. Thus, $\Gamma$ is the only variable in Eq. (21) that depends on the induction at the section:

$$
\begin{aligned}
\Gamma & =\frac{\Gamma_{\mathrm{dyn}}}{\Gamma_{\mathrm{QS}}} \frac{1}{2} c C_{\mathrm{L}} v_{\mathrm{r}}, \\
& =\frac{\Gamma_{\mathrm{dyn}}}{\Gamma_{\mathrm{QS}}} c \pi v_{\mathrm{r}} \alpha, \\
& =\frac{\Gamma_{\mathrm{dyn}}}{\Gamma_{\mathrm{QS}}} c \pi \sqrt{\left(v_{\infty}-W_{i-1}\right)^{2}+(\Omega r)^{2}} \arctan \left(\frac{v_{\infty}-W_{i-1}}{\Omega r}\right),
\end{aligned}
$$

where $v_{\infty}$ is the free wind speed. The step response function from Eq. (15) evaluated at half a time step approximates $\Gamma_{\mathrm{dyn}} / \Gamma_{\mathrm{QS}}$ because we consider a buildup of the circulation from zero.

Equation (21) is plotted for different time steps as a function of $W_{i-1}$, the induction from the previous iteration, for the blade tip section of the NREL $5 \mathrm{MW}$ reference turbine at $8 \mathrm{~m} \mathrm{~s}^{-1}$ wind speed and $9.2 \mathrm{rpm}$ rotor speed, corresponding to a tip speed ratio of 7.6, in Fig. 8. The airfoil camber is neglected.

The intersections of the curves with the blue curve $\left(W_{i}=\right.$ $\left.W_{i-1}\right)$ are the converged solutions, where a new iteration would lead to exactly the same induction as the previous one. The calculations for different time steps have different converged inductions, because the length of the trailed vortex filaments is proportional to the time step. However, not only the converged solution but also the gradient of the curves changes, which leads to a condition for convergence: if the distance from the converged solution decreases during a time step,

$\left|W_{i}-W_{\text {conv }}\right|<\left|W_{i-1}-W_{\text {conv }}\right|$, the iterative process converges. As seen in Fig. 8, the gradient of the curves is almost independent of $W_{i-1}$. The gradients are negative because induction reduces the angle of attack. Therefore, an approximation of condition (25) can be used:

$$
\frac{\mathrm{d} W_{i}}{\mathrm{~d} W_{i-1}}>-1 \text {. }
$$

This gradient can be derived from Eqs. (21) and (24) as

$$
\begin{aligned}
& \frac{\mathrm{d} W_{i}}{\mathrm{~d} W_{i-1}}=\frac{\Gamma_{\mathrm{dyn}}}{\Gamma_{\mathrm{QS}}} \pi c\left(B_{1}-B_{2}\right) \\
& \left(\frac{\alpha\left(v_{\infty}-W_{i-1}\right)}{v_{\mathrm{r}}}+\frac{v_{\mathrm{r}}}{\Omega r\left(\left(\frac{v_{\infty}-W_{i-1}}{\Omega r}\right)^{2}+1\right)}\right),
\end{aligned}
$$

where

$$
B_{v}=(-1)^{v}\left(D_{X, v}\left(1-e^{-\Delta \beta / \Phi_{v}}\right)+D_{Y, v}\left(1-e^{-4 \Delta \beta / \Phi_{v}}\right)\right) .
$$

The gradient is mainly depending on the time step and point density (through $B_{1}$ and $B_{2}$ ) and the rotational speed.

Instead of reducing time step and point density until a simulation is stable, which can lead to time steps orders of magnitude smaller than commonly used in aeroelastic codes and low spatial resolution, a relaxation factor $f_{\mathrm{r}}$ can be introduced, so that

$W_{i, \mathrm{r}}=W_{i}\left(1-f_{\mathrm{r}}\right)+W_{i-1} f_{\mathrm{r}}$.

The derivative of this downwash with regard to the old downwash is

$\frac{\mathrm{d} W_{i, \mathrm{r}}}{\mathrm{d} W_{i-1}}=\frac{\mathrm{d} W_{i}}{\mathrm{~d} W_{i-1}}\left(1-f_{\mathrm{r}}\right)+f_{\mathrm{r}}$.

For the minimum relaxation factor $f_{\mathrm{r}}$, which allows for a stable computation $\left(\mathrm{d} W_{i, \mathrm{r}} / \mathrm{d} W_{i-1}=-1\right)$, follows

$f_{\mathrm{r}}=-\frac{1+\frac{\mathrm{d} W_{i}}{\mathrm{~d} W_{i-1}}}{1-\frac{\mathrm{d} W_{i}}{\mathrm{~d} W_{i-1}}}$,

which can be determined depending on the time step $\Delta t$, the point distribution, and the number of points on the blade.

In the initial phase of the simulation, the maximum relaxation factor for all blade sections can be quickly determined by setting $W_{i-1}=0$ in Eq. (27) and looping through the sections. The highest necessary relaxation factor for one section that has been found is then used for the axial and tangential induction on the whole blade. As the simulation continues, the relaxation factor can be updated whenever there are large changes in rotational speed, induction, or blade pitch. If the relaxation factor is updated every several time steps, then determining the relaxation factor takes negligible computation time. Choosing a slightly more conservative relaxation factor than what has been estimated will also ensure stability in different conditions than the ones the factor was based on. 


\section{Accelerating the NWM}

In this section, an approach to accelerate the model is presented. The number of exponential terms used to approximate the decreasing induction with increasing distance from the blade in Eq. (1) is reduced to one. Using only one exponential term removes the $Y_{\mathrm{w}}$ component in the near-wake algorithm, Eq. (3c) and thus halves the computation time.

The reduced approximation function is defined as

$$
\frac{\mathrm{d} w}{\mathrm{~d} w_{0}} \approx 1.359 e^{-\beta / \Phi}-0.359 e^{-4 \beta / \Phi} \approx A^{*} e^{-\beta / \Phi^{*}} .
$$

The values of $A^{*}$ and $\Phi^{*}$ are found by solving the following equations:

$$
\begin{aligned}
& W(\beta=\infty)=\int_{0}^{\infty} 1.359 e^{-\beta / \Phi}-0.359 e^{-4 \beta / \Phi} \mathrm{d} \beta \\
& =\int_{0}^{\infty} A^{*} e^{-\beta / \Phi^{*}} \mathrm{~d} \beta, \\
& \int_{0}^{\infty} W(\beta=\infty)-W(\beta) \mathrm{d} \beta \\
& =\int_{0}^{\infty} \Phi\left(1.359 e^{-\beta / \Phi}-\frac{0.359}{4} e^{-4 \beta / \Phi}\right) \mathrm{d} \beta \\
& =\int_{0}^{\infty} \Phi^{*}\left(A^{*} e^{-\beta / \Phi^{*}}\right) \mathrm{d} \beta .
\end{aligned}
$$

Equation (33) ensures that the quasi-steady induction $W(\beta=$ $\infty$ ) of the reduced model is equal to the one computed by the original model for a trailed vortex with constant strength. Equation (34) ensures a good dynamic behavior by requiring the time integral of the difference between dynamic and quasi-steady induction to be identical to the original model. The solution to these equations is

$A^{*}=\frac{(1.359-0.359 / 4)^{2}}{1.359-0.359 / 16}, \Phi^{*}=\Phi \frac{1.359-0.359 / 16}{1.359-0.359 / 4}$

A comparison of the buildup of induction in time, corresponding to the integral of the exponential functions, is shown in Fig. 9. The largest deviations of the reduced model from the original model are below $2.5 \%$ of the quasi-steady induction $W(\beta=\infty)$.

\section{Free-wake code}

GENUVP is a potential flow solver combining a panel representation of the solid boundaries (blades) with a vortex particle representation of the wake. In the present work, the

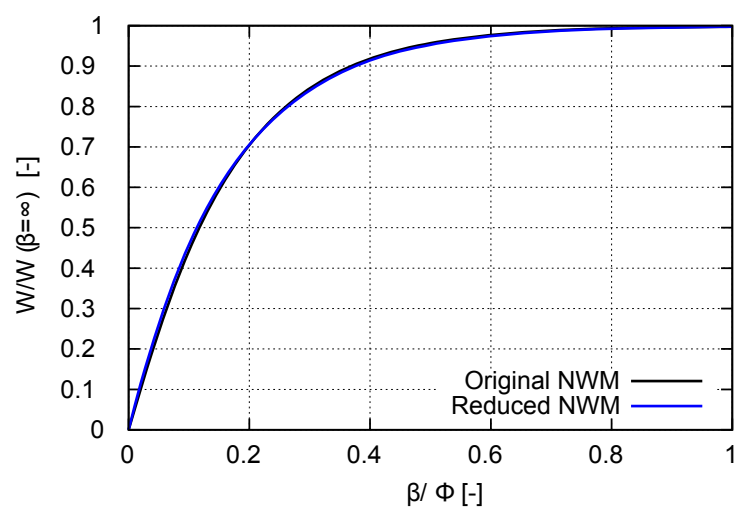

Figure 9. Comparison of induction buildup between full NWM and reduced NWM, depending on the length of a trailed vortex filament with constant circulation.

blades are considered as thin lifting surfaces carrying piecewise constant dipole distribution (equivalent to horseshoetype vortex filaments). Blades shed vorticity in the wake along their trailing edges and their tips (vorticity emission line). In the model a hybrid wake approach is followed, which refers to the mixed formulation used in the representation of the wake. In this formulation, the dipole representation is retained for the near part (equivalent to horseshoe filaments), while the far part is modeled by free vortex particles. The near-wake part, consisting of the newly shed vorticity trailed within the current time step, is modeled as a vortex sheet also carrying piecewise constant dipole distribution. Within every time step, a strip of wake panels is released that are in contact with the emission line. By applying the no-penetration boundary condition at the center of each solid panel and the Kutta condition along the emission line, the unknown dipole intensities are determined. Then, at the end of each time step, the newly shed vorticity is transformed into vortex particles and all vortex particles are convected downstream with the local flow velocity (free-wake representation) into their new positions. The layout of the modeling is shown in Fig. 10. Details of the model can be found in Voutsinas (2006).

Since GENUVP is defined as a potential flow solver, the loads need correction in order to account for viscous effects. This is done by means of the generalized ONERA unsteady aerodynamics and dynamic stall model (Petot, 1989). The potential load is calculated by integrating pressures (pressure differences between pressure and suction side) over the lifting surfaces. Then, viscous corrections are applied to the potential sectional loads that require the local flow velocity and angle of attack at every section as input. In particular, the ONERA model splits the aerodynamic forces into a potential and a separated flow component. This is done through the introduction of two equivalent circulation parameters defined both for the lift and the drag force. In GENUVP and in the case of attached flow conditions, no correction is applied on 


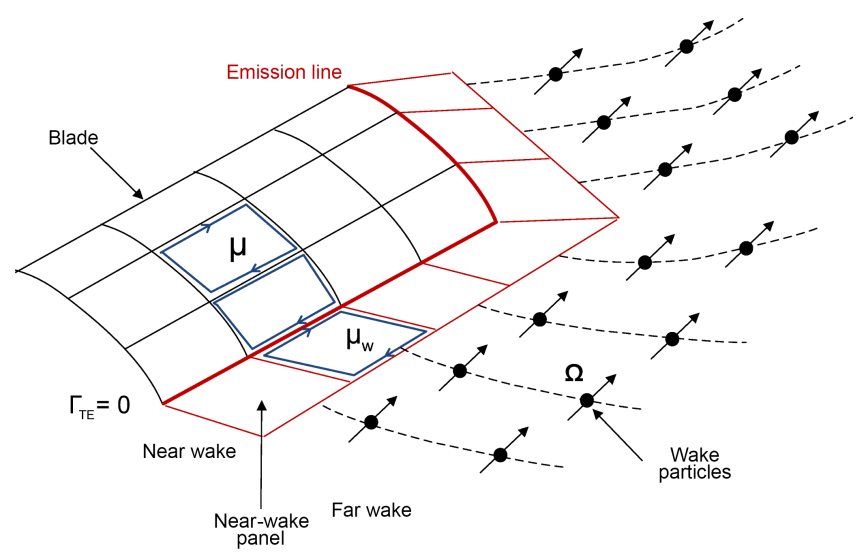

Figure 10. Layout of the free-wake modeling of a blade: black lines define the blade surface panels, red lines define the wake generated within a time step, and symbols represent freely moving particles.

the unsteady lift force computed by the free-wake code. The only correction applied is the inclusion of the viscous drag contribution to the loads. In the case of separated flow conditions the separated flow component of the ONERA model is superimposed to the potential loads provided by the freewake model (Riziotis and Voutsinas, 1997).

In the case of a flexible blade, flow equations are solved for the deformed blade geometry, while deformation velocities are accounted for in formulating the non-penetration boundary condition.

The GENUVP free-wake code has been thoroughly validated over the past years against measured data both on wind turbines and helicopter rotors in the framework of numerous EU-funded projects. Blade loads and wake velocities comparisons against measurements have been performed on the MEXICO rotor in the context of Innwind.eu project (Madsen et al., 2015). Moreover, detailed blade load calculations have been performed for the NREL test rotor and results have been compared to experimental data (NREL experiment) and computational fluid dynamics computations (Chassapoyiannis and Voutsinas, 2013). Extensive validation of the code has been also performed in the framework of the HeliNovi project, where aerodynamic and structural loads, wake velocities and elastic deflections have been compared to tunnel measurements on a BO105 helicopter model (Dieterich et al., 2005).

\section{Results}

In the following section, the effectiveness of the iteration procedure and the estimation of the relaxation factor are demonstrated for a horseshoe vortex. Then, in Sect. 8.2, the unsteady induction predicted by the coupled near- and farwake model is compared with results from an unsteady BEM model and the free-wake code described in Sect. 7. Pitch steps and prescribed vibrations of the blades of the NREL $5 \mathrm{MW}$ reference turbine are investigated.

\subsection{Iteration procedure}

To illustrate the efficiency of the iterative implementation, induction buildups for a simplified case are shown in Fig. 11. The simple test case is a wing with a span of $0.3 \mathrm{~m}$ and a constant bound circulation, so that only two vortices with opposite vortex strength are trailed at the edges. To use the NWM and ensure parallel flow, the wing is modeled as the only aerodynamic section at the end of a $10 \mathrm{~km}$ long blade. The free stream velocity is $70 \mathrm{~m} \mathrm{~s}^{-1}$. At $t=1 \mathrm{~s}$, the geometric AOA of the wing with a symmetrical profile is increased from 0 to $5^{\circ}$ within $0.02 \mathrm{~s}$. The lift coefficient is $2 \pi \alpha$ and the chord $1 \mathrm{~m}$. The left side of Fig. 11 shows the induction buildup for different time steps without iterating, while the right side shows the effect of the iteration procedure. Both the overshoot of the induction for a time step of $0.002 \mathrm{~s}$ and the oscillations for a time step of $0.02 \mathrm{~s}$ are reduced by the iteration procedure.

The relaxation factors estimated as proposed in Sect. 5.2 are compared with the lowest stable relaxation factors obtained by trial and error in Fig. 12 for the NREL $5 \mathrm{MW}$ reference turbine operating at 8 and $25 \mathrm{~m} \mathrm{~s}^{-1}$ wind speed in uniform inflow. The comparison in Fig. 12 shows that the estimated relaxation factor is conservative, but the safety margin towards unstable computation is smaller in the $25 \mathrm{~m} \mathrm{~s}^{-1}$ case.

\subsection{Comparison of the coupled model with a BEM model and a free-wake panel code}

In the following Sect. 8.2.1, the main differences between the coupled near- and far-wake model and the free-wake code are described. It is also highlighted how the validation cases in the following sections (predicted force responses to pitch steps, Sect. 8.2.2; blade vibrations, Sect. 8.2.3) are chosen to investigate the effects of the various model differences. All computations use the refined blade model shown in Fig. 6. The blade has been discretized using 40 radial aerodynamic stations in the BEM-based codes, with corresponding 41 vortices in the coupled model trailed from root, tip and in between stations. For the lifting-surface free-wake simulations, the blade has been discretized using 35 spanwise and 11 chordwise grid lines. Compared to the faster models, the resolution was mainly reduced close to the blade root.

\subsubsection{Inherent differences between coupled near- and far-wake model and free-wake code}

This paragraph contains an overview of the inherent modeling differences between the different models. It is also detailed how these differences are investigated in the following comparisons of aerodynamic response to pitch steps and prescribed blade vibrations. 

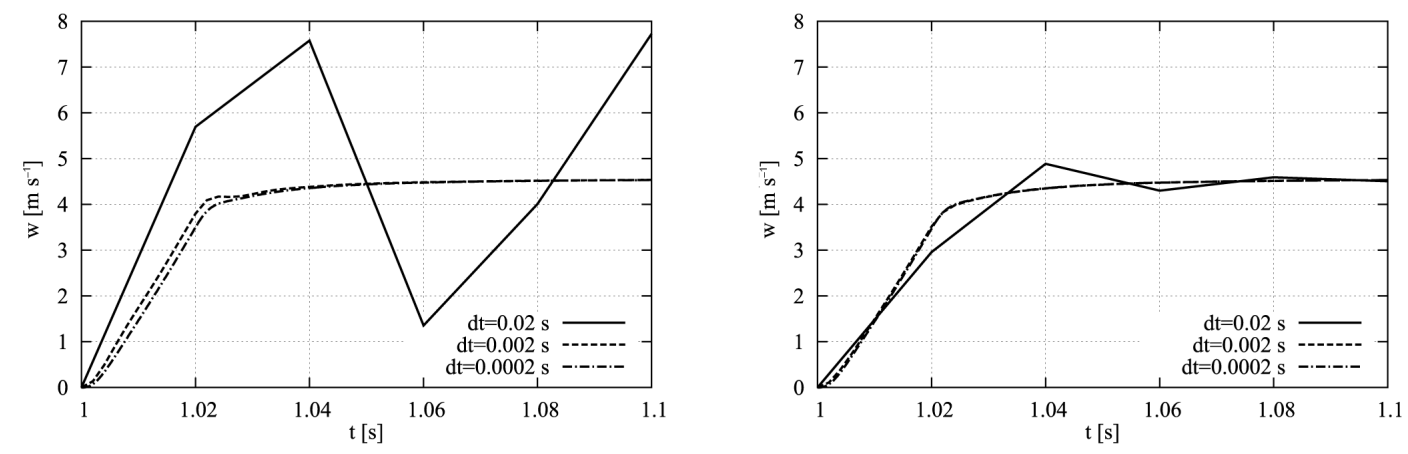

Figure 11. Buildup of the downwash for a horseshoe vortex depending on the time step. The NWM tends to be unstable (left) but can be stabilized by iterating to convergence of the downwash (right).
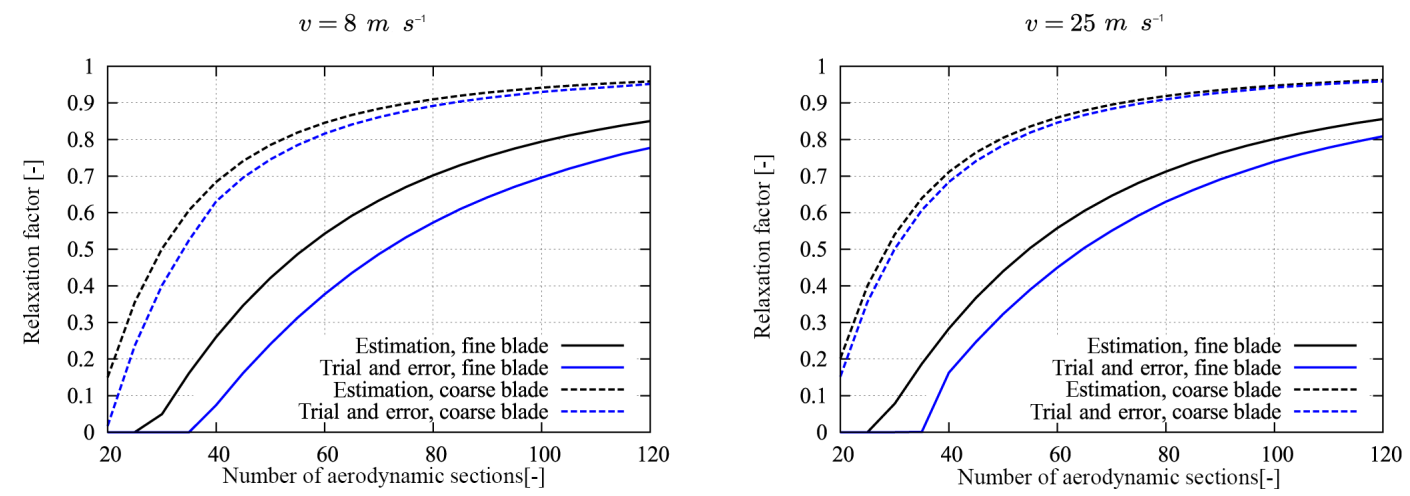

Figure 12. Estimated relaxation factor compared with the lowest stable relaxation factor from trial and error depending on the number of aerodynamic sections. The time step is $0.02 \mathrm{~s}$. The estimated relaxation factors, Eq. (31), are conservative and the influence of the refined blade geometry is captured.

The free-wake code uses a lifting-surface approach, while the near-wake model uses a lifting-line approach. The freewake code models the airfoils as the camber line of the airfoil sections (thin airfoil approach) using potential theory. Thereby, the different sections of the blade have a lift gradient of $2 \pi$ and zero drag. Drag and the lift gradient deviation from $2 \pi$ is then added afterwards as part of the ONERA unsteady airfoil aerodynamics model. The faster models, on the other hand, all use the airfoil data directly. Thus, the measured lift gradients are used for both the shed vorticity model and the near-wake model. The influence of this difference is evaluated in the vibration comparisons section by setting the lift gradient to $2 \pi$ and drag to zero in the case of an edgewise vibration, where drag contributes significantly to the aerodynamic work.

The time constants for the shed vorticity model in the BEM-based codes do not contain a correction for airfoil thickness but are instead the approximations for a flat plate originally obtained by Jones. The flat plate approximation agrees with the thin lifting surface in the free-wake simulations. The comparisons of the aerodynamic response to prescribed blade vibrations contain also results from a BEM model with deactivated shed vorticity model. These results are included to evaluate the isolated influence of the shed vorticity modeling and distinguish the dynamic effects of shed and trailed vorticity. In the free-wake code, the shed vorticity is inherently modeled and can not be turned off. In contrast to the fast dynamic effects due to trailed and shed vorticity close to the blade, there are also slow dynamic inflow effects. This term is used here to describe effects that would also be visible in actuator disk simulations where the individual blades are not modeled at all. The slow dynamic effects are modeled directly in the free-wake code and by means of a dynamic inflow model in the other codes. The influence of these effects is compared for pitch steps where the free-wake code results directly model the influence of wake expansion. In the case of the blade vibrations, on the other hand, the main effects occur in the direct wake close to the blade. The influence of dynamic inflow in these cases is very small, which is reflected in the large time constants in the modeling. Wake expansion is also expected to be of minor importance in these cases.

Another difference between the free-wake code and the BEM-based models is that the dynamic interaction between a blade and the wake of the other blades is only modeled in the free-wake code. This introduces dynamic variations in the 

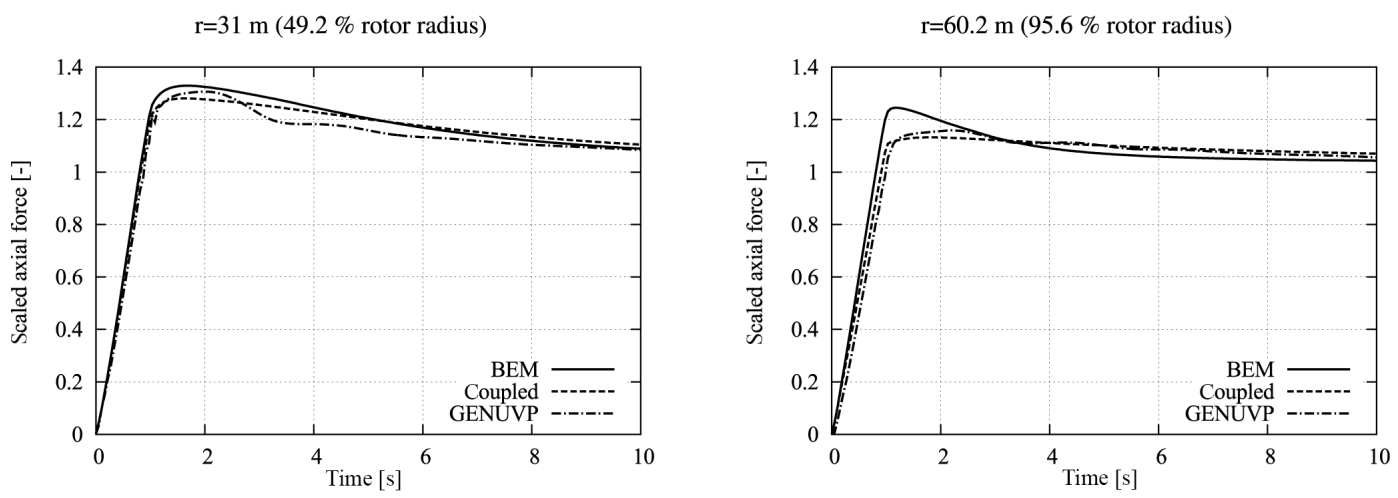

Figure 13. Scaled axial force at different radial positions during and after a pitch step by $5^{\circ}$ in $1 \mathrm{~s}$.
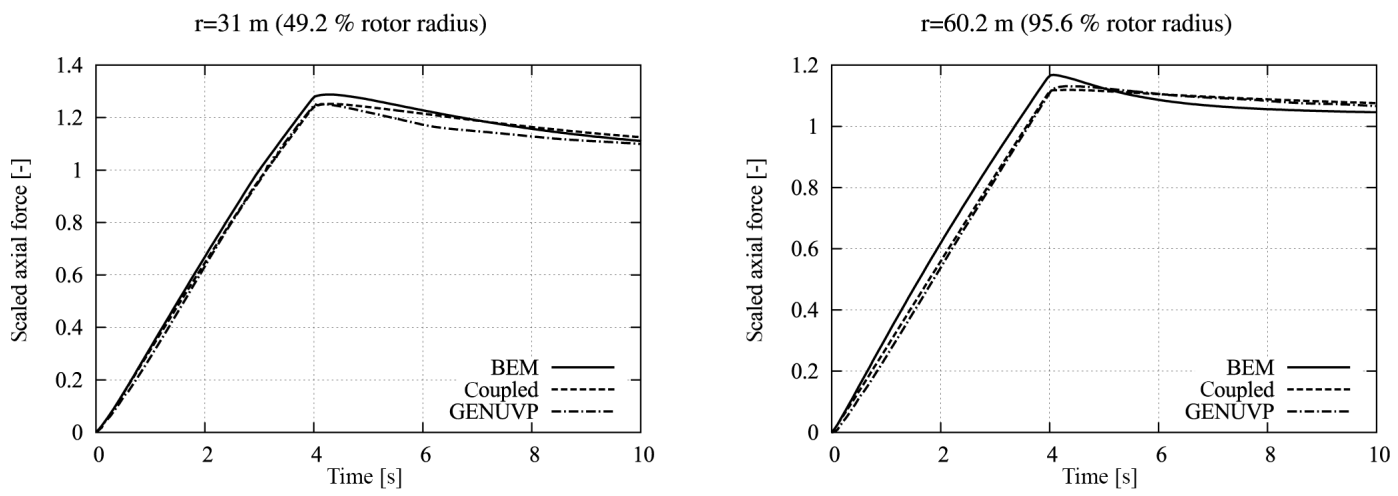

Figure 14. Scaled axial force at different radial positions during and after a pitch step by $5^{\circ}$ in $4 \mathrm{~s}$.

pitch step cases that are missing in the BEM-based codes. If this blade-wake interaction were to play an important role in the vibration cases, the agreement between the codes should be better in the high wind speed cases with a larger helix angle than in the low wind cases, where the wake of previous blades is closer to the rotor plane when a blade is passing.

In order to avoid additional uncertainties due to dynamic stall modeling, all cases have been chosen such that stall is mostly avoided. To obtain this, the pitch steps are conducted from $5^{\circ}$ to feather towards normal operation, and the amplitudes in the vibration cases are generally small. There may be stall at the very root in the prescribed vibration cases, but the amplitude there is almost zero. Thus, there is very little contribution from the root section to the aerodynamic work and differences in dynamic stall modeling do not visibly change the results.

\subsubsection{Pitch steps}

Pitch steps with stiff blades have been performed where the NREL $5 \mathrm{MW}$ reference turbine is operating at a wind speed of $8 \mathrm{~m} \mathrm{~s}^{-1}$ and a rotation speed of $9.2 \mathrm{rpm}$. The turbine starts with blades that are pitched by $5^{\circ}$ to feather. The time steps are $0.054 \mathrm{~s}$ (120 steps per revolution) for GENUVP and $0.05 \mathrm{~s}$ for the other models. After $60 \mathrm{~s}$ simulated time, the blades are pitched to $0^{\circ}$ at constant pitch rate in 1 or $4 \mathrm{~s}$. The forces are normalized to compare the dynamics of the pitch response, such that the force before the pitch step is 0 and the force $45 \mathrm{~s}$ after the pitch step is 1 .

Figure 13 show the axial force response at a position at mid-blade and close to the blade tip for the fast pitch step. The free-wake code predicts a slower force response during the pitch step than the BEM model. The results of the coupled model during the pitch step lie in between the other codes. In the free-wake code results, some oscillations are present after the pitch step, especially at the mid-blade section. The oscillations occur at a 3P frequency and are likely due to the individual blades passing by the trailed vortex wakes of the other blades. These oscillations make it difficult to judge whether the BEM model or the coupled model is predicting the overshoot closer to the free-wake code, the results of which are in between the two.

The results of the slower pitch step in Fig. 14 show less oscillations of the free-wake code results. In this case, the predicted results from the coupled model clearly agree better with the free-wake code than the BEM results, both on the slope during the pitching motion and on the predicted overshoot. 

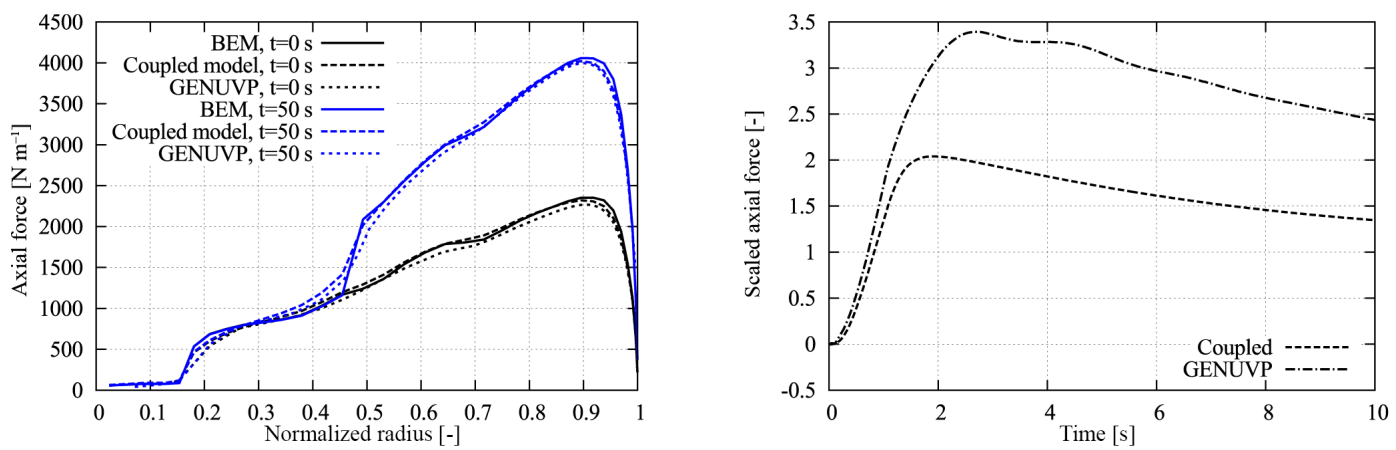

Figure 15. Left plot: force distribution before the partial pitch step and $50 \mathrm{~s}$ after. Right plot: time history of the axial force comparing coupled model and GENUVP at $21.6 \mathrm{~m}$ radius (34.3\% rotor radius). The BEM gives a constant force.

Axial force distributions for a partial pitch comparison at $8 \mathrm{~m} \mathrm{~s}^{-1}$ are shown in Fig. 15. In this case, only the outer half of the blade is pitched from 5 to $0^{\circ}$ over $1 \mathrm{~s}$. As shown in the left plot of Fig. 15, the trailed vorticity effect at the midblade is predicted by both the coupled aerodynamics model and the free-wake code to a similar degree. The effect looks like a radial smoothing of the loading, because the trailed vorticity increases the angle of attack inboard of the pitched blade half and decreases the angle of attack at the beginning of the higher loaded outer half of the blade. In the right plot of Fig. 15, the time history of the axial force is compared at $34.3 \%$ radius. The constant force predicted by the BEM model on this non-pitching part of the blade is not included in this comparison. The overshoot is underpredicted by the coupled model by around $40 \%$. The induction predicted by the coupled model stops increasing after $1.6 \mathrm{~s}$, corresponding to a quarter revolution at $9.2 \mathrm{rpm}$, while the force predicted by GENUVP continues to increase. Figure 15 thus illustrates that the coupled model can predict a change in loading that can not be computed based on BEM theory, but the restriction to a quarter revolution is limiting in this case. The difference in the overshoot prediction at $21.6 \mathrm{~m}$ radius amounts to roughly $70 \mathrm{~N} \mathrm{~m}^{-1}$.

\subsubsection{Prescribed vibrations}

The aerodynamic response to blade vibrations is investigated for normal operation at 8 and $25 \mathrm{~m} \mathrm{~s}^{-1}$. The corresponding rotor speeds are 9.2 and $12.1 \mathrm{rpm}$ and the pitch angles 0 and $23.2^{\circ}$, respectively. The force response is compared in terms of radial distributions of aerodynamic work during one oscillation, where a positive aerodynamic work corresponds to a positive aerodynamic damping of the vibration. The mode shapes are chosen as the first and second structural mode shapes of the NREL $5 \mathrm{MW}$ reference turbine blade at standstill (see Fig. 16). To simplify the comparison, the vibrations have been prescribed as collective in-plane or out-of-plane vibrations. The frequencies, amplitudes and time steps used for the computations are shown in Table 1, as well as the modal masses that are used for damping estimations. For the

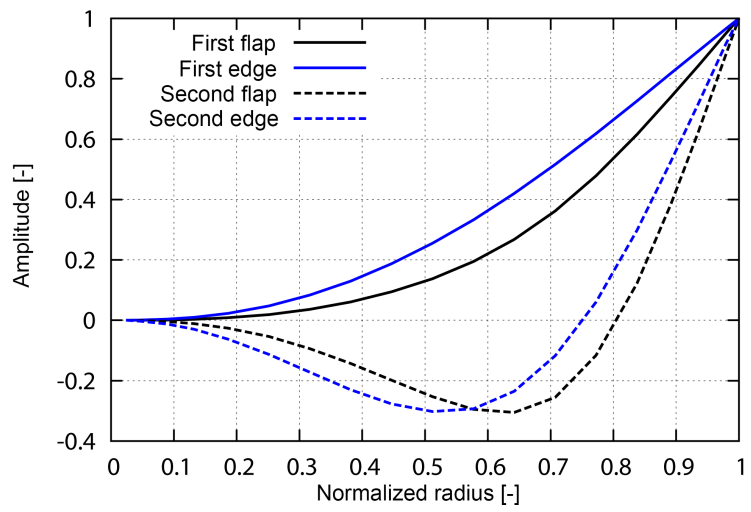

Figure 16. Mode shapes used in the work computations, which are simplified to be purely in-plane or out-of-plane deflections.

Table 1. Modal parameters and time steps prescribed in the work comparison. The time steps for the first flap were chosen as 180 steps per revolution and thus depend on the rotor speed.

\begin{tabular}{lccccc}
\hline Mode & $\begin{array}{c}f \\
{[\mathrm{~Hz}]}\end{array}$ & $\begin{array}{c}m \\
{[\mathrm{~kg}]}\end{array}$ & $\begin{array}{c}A \\
{[\mathrm{~m}]}\end{array}$ & $\begin{array}{c}\mathrm{d} t \\
{[\mathrm{~s}]}\end{array}$ \\
\hline First flap & 0.66 & 905 & 0.25 & 0.5 & $0.036,0.028$ \\
First edge & 1.0 & 1480 & 0.25 & 1.0 & 0.025 \\
Second flap & 1.82 & 594 & 0.25 & 0.01 \\
Second edge & 3.2 & 793 & 0.25 & 0.01 \\
\hline
\end{tabular}

first modes only results at the larger amplitudes are shown in the following. Investigating the results at the smaller amplitudes leads to the same conclusions.

In the BEM and coupled model, the blade section velocities due to the vibrations are added to the relative wind speed. The deflection of the blade and the resulting change in the section positions have been neglected because the amplitudes are small compared to the blade radius. In the free-wake code, not only deflection velocities but also deformation of the blade shape is considered. 

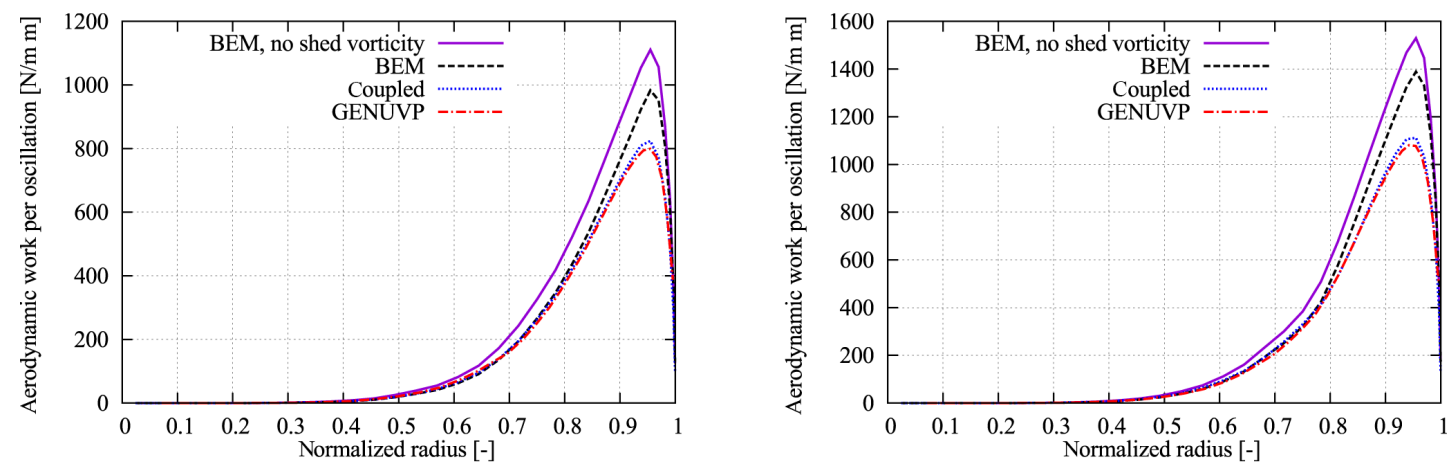

Figure 17. Aerodynamic work per oscillation of first flap motion at $8 \mathrm{~m} \mathrm{~s}^{-1}$ (left) and $25 \mathrm{~ms}^{-1}$ (right) wind speed with an amplitude of $0.5 \mathrm{~m}$.
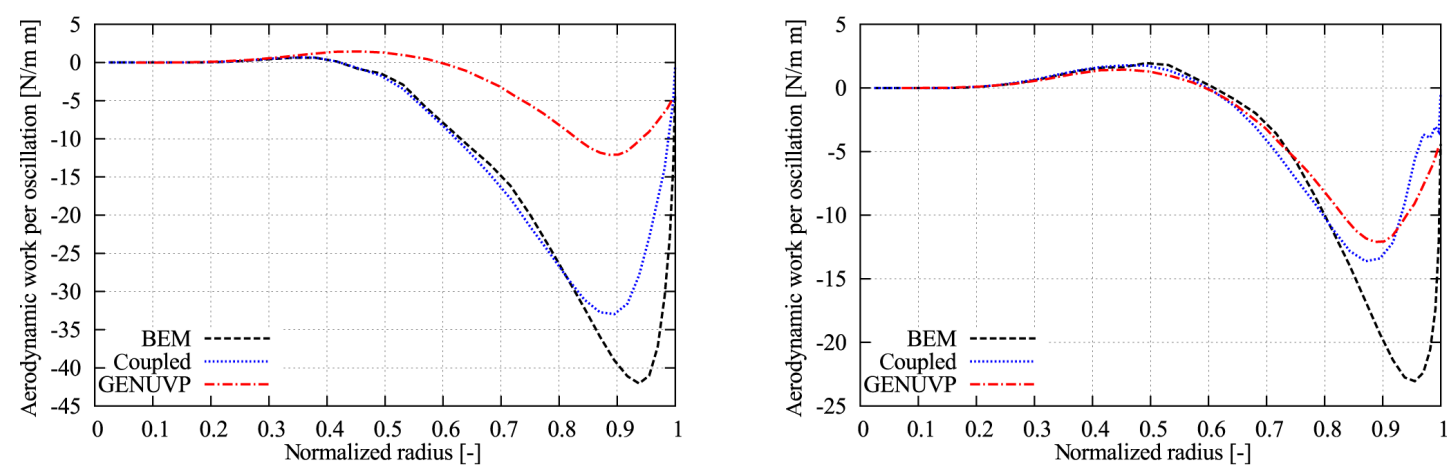

Figure 18. Aerodynamic work per oscillation of first edge motion at $8 \mathrm{~m} \mathrm{~s}^{-1}$ at an amplitude of $1 \mathrm{~m}$. Drag has been excluded, and $C_{\mathrm{L}}=2 \pi \alpha$. In the left plot, $\alpha_{\mathrm{QS}}$ is used in the unsteady airfoil aerodynamics model in both coupled model and BEM (see Eqs. 8-11). In the right plot, the unsteady airfoil aerodynamics model uses $\alpha_{\mathrm{QS}}-\alpha_{0}$ (see Eq. 20), improving the agreement with the free-wake code results.

To distinguish the effects of shed and trailed vorticity, the following comparisons include a BEM computation with a disabled shed vorticity model. The aerodynamic work during out-of-plane motion according to the first flap mode shape is shown in Fig. 17 for 8 and $25 \mathrm{~m} \mathrm{~s}^{-1}$. The work integrated over the blade is overpredicted by the BEM model (dashed black line) by about $10 \%$ compared to the free-wake code in both cases. If the three BEM-based results are compared, it shows that the trailed vorticity (dotted blue line versus dashed black line) has an influence of the same order of magnitude as the shed vorticity (solid purple versus dashed black line). The trailed vorticity effects are more important close to the tip vortex, while the influence of the shed vorticity extends across the whole blade. The results of the coupled model are very close to the free-wake code results but deviate slightly towards higher work. The influence of the trailed vorticity behind the other two rotor blades, which is not included in the NWM, on the vibration response is found to be small compared to the influence of the wake of the blade itself in normal operation.

The in-plane vibrations at $8 \mathrm{~ms}^{-1}$ are almost parallel to the inflow and the drag forces contribute much more to the work than in the other cases. To simplify the problem, drag has been excluded from the aerodynamic work computations presented in Fig. 18. Further, the lift gradient has been assumed as $2 \pi$. In the left plot of Fig. 18, the quasi-steady angle of attack is used in the unsteady airfoil aerodynamics model (see Eqs. 8-11). The agreement in this case is poor. In the right plot, the zero lift angle due to camber is included in the quasi-steady angle of attack (see Eq. 20). This approach leads to a much improved result for the BEM-based codes and good agreement of the coupled model and GENUVP.

With the airfoil polars of the NREL $5 \mathrm{MW}$ reference turbine the agreement between the codes is not as good in the $8 \mathrm{~m} \mathrm{~s}^{-1}$ case; see the left plot of Fig. 19.

However, the coupled model produces results much closer to the free-wake code close to the blade tip than the BEM model. At $25 \mathrm{~m} \mathrm{~s}^{-1}$, where the work is predominantly due to the vibration component perpendicular to the inflow as a result of blade pitch, the coupled near- and far-wake model agrees similarly well with the free-wake code as in the cases with out-of-plane vibrations discussed above. The shed vorticity effects on the in-plane vibrations are larger than on the out-of-plane vibrations due to the higher frequency and the larger relative velocity variations.

Figure 20 shows results for the second modes. 

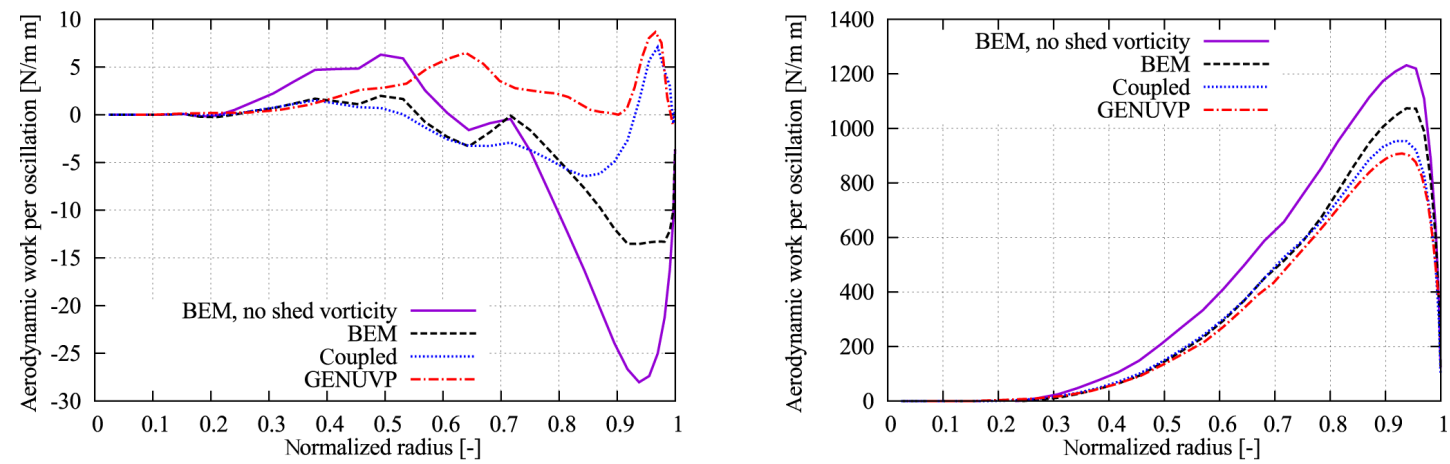

Figure 19. Aerodynamic work per oscillation of first edge motion at $8 \mathrm{~m} \mathrm{~s}^{-1}$ (left) and $25 \mathrm{~m} \mathrm{~s}^{-1}$ (right) wind speed with an amplitude of $1.0 \mathrm{~m}$.
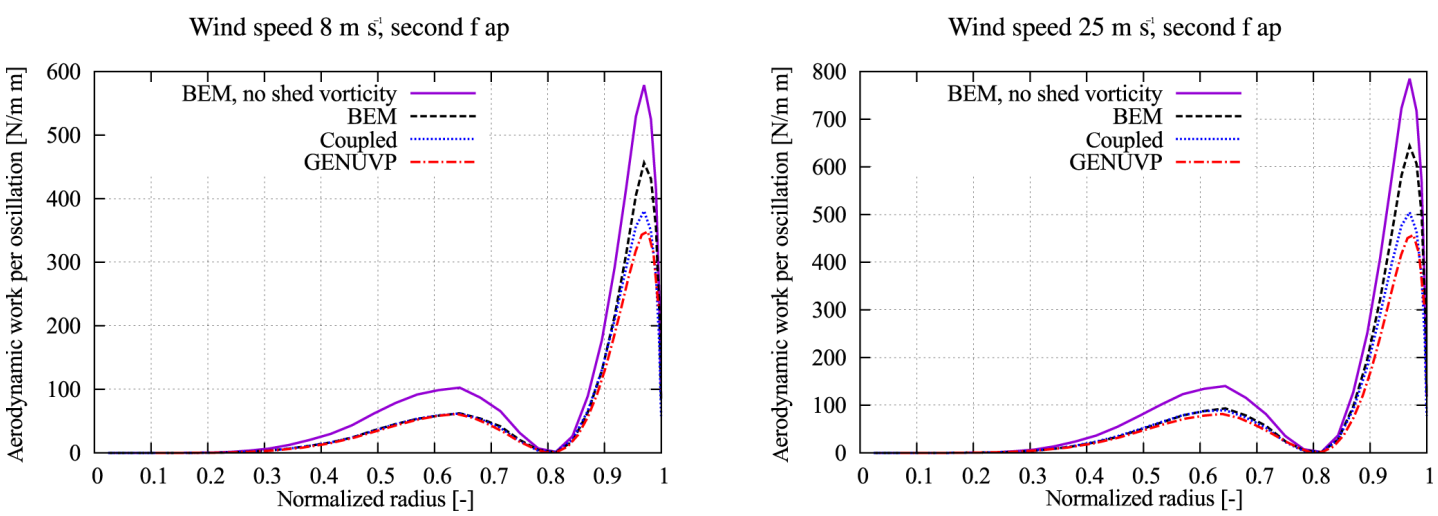

Wind speed $8 \mathrm{~m} \mathrm{~s}^{-1}$, second edge
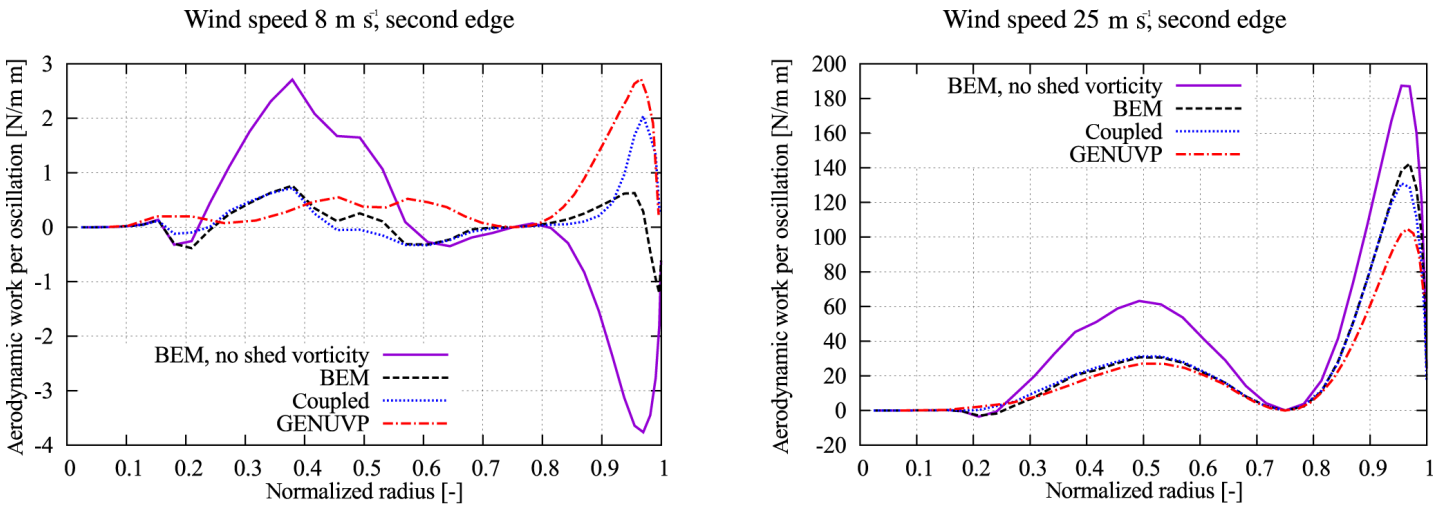

Figure 20. Aerodynamic work per oscillation of second flap (top) and edge (bottom) motion at $0.25 \mathrm{~m}^{-1 m p l i t u d e}$ at $8 \mathrm{~ms}^{-1}$ (left) and $25 \mathrm{~m} \mathrm{~s}^{-1}$ (right).

The BEM model results compare similarly well with the GENUVP results as for the first modes. Because the frequencies of the second modes are higher the shed vorticity model is more important in these cases. The importance of the trailed vorticity model at higher frequencies does not increase by the same amount, because the higher reduced frequencies affect the buildup of the unsteady bound circulation (see Eq. 18). The coupled model results are closer to the free-wake results than the BEM results in all cases, but as opposed to the comparisons above, the coupled model is un- derestimating the effects of the dynamics of the tip vortex. Further inboard the free-wake code predicts slightly lower aerodynamic work in the $25 \mathrm{~m} \mathrm{~s}^{-1}$ case than the BEM model, which can not be seen in the coupled model results. Also, the agreement of the coupled model and GENUVP is worse in the edgewise case than in the flapwise case at $25 \mathrm{~m} \mathrm{~s}^{-1}$, which has not been seen to that extend for the first edgewise cases (see Figs. 17 and 19). A reason for this might be that the second edgewise case is computed with fewer time steps per period of oscillation to limit the computational cost. 
For easier evaluation of the force response differences, the aerodynamic work can be expressed in terms of a damping ratio of a respective blade mode. Because the computations have been based on prescribed purely in-plane and out-ofplane structural mode shapes, these dampings do not correspond to any aeroelastic blade modes. For a system with a single degree of freedom with the modal mass $m$ and frequency $f$, given in Table 1 , the damping ratio $\xi$ and logarithmic decrement $\delta$ are

$\xi=\frac{W_{\text {aero }}}{8 \pi^{3} A^{2} f^{2} m}=\frac{1}{\sqrt{1+\left(\frac{2 \pi}{\delta}\right)^{2}}}$,

where $A$ is the amplitude and $W_{\text {aero }}$ the aerodynamic work per oscillation period. The estimated logarithmic decrements according to Eq. (36) corresponding to the first flap motion at $8 \mathrm{~m} \mathrm{~s}^{-1}$ with an amplitude of $0.5 \mathrm{~m}$ (see Fig. 17) are $334 \%$ for the BEM results, $300 \%$ for the coupled model and $292 \%$ for the free-wake code results. Flapwise modes are highly damped and thus these changes in the damping will not significantly alter the blade fatigue loads. On the other hand, lower aerodynamic damping of flapwise blade motion will correspond to a lower aerodynamic damping of tower foreaft motion and might thus lead to increased tower fatigue loads. It is expected that this lower aerodynamic damping is balanced to some degree because the near-wake effects reduce the aerodynamic excitation due to atmospheric turbulence.

Aerodynamic damping estimations for the first in-plane vibrations at $8 \mathrm{~ms}^{-1}$ at $1 \mathrm{~m}$ amplitude are shown in Table 2 . The damping has been estimated in four cases, which differ in the airfoil polars and the modeling of the camber effect on the unsteady airfoil aerodynamics. Comparison of the first two cases of Table 2 shows that the induced drag caused by airfoil camber in the shed vorticity modeling results in a damping of roughly $0.7 \%$ logarithmic decrement. According to the BEM and coupled model results in cases (3) and (4), the airfoil drag increases the logarithmic decrement by about $0.3 \%$. The trailed vorticity decreases the absolute value of the damping by roughly $0.14 \%$ log decrement. Further, comparing columns (2) and (4), the combined influence of airfoil polars with lift coefficients other than $2 \pi$ and non-zero drag is close to 3 times larger in the free-wake code computations, which is caused by the different unsteady drag modeling. The small differences in estimated logarithmic decrement can have an impact on loads and stability computations for edgewise modes with a very low aeroelastic damping.

In the out-of-plane prescribed vibration cases investigated, the trailed vorticity reduces the aerodynamic work. Further, a previous study by Pirrung et al. (2014) showed that the trailed vorticity effects will delay the onset of flutter towards higher rotor speeds. This is in agreement with findings on the influence of shed vorticity, which leads to both a decrease of the flapwise damping and increased flutter speeds of a vibrating 2-D blade section (Hansen, 2007).
Table 2. Estimated logarithmic decrements [\%] corresponding to the aerodynamic work of first in-plane vibrations at $8 \mathrm{~m} \mathrm{~s}^{-1}$, based on the results at an amplitude of $1 \mathrm{~m}$.

\begin{tabular}{lcccc}
\hline & $(1)$ & $(2)$ & $(3)$ & $(4)$ \\
& $\begin{array}{c}C_{\mathrm{L}}^{\prime}=2 \pi, \\
C_{D}=0, \\
\text { no camber }\end{array}$ & $\begin{array}{c}C_{\mathrm{L}}^{\prime}=2 \pi, \\
C_{\mathrm{D}}=0\end{array}$ & $\begin{array}{c}\text { NREL } C_{\mathrm{L}}, \\
C_{\mathrm{D}}=0\end{array}$ & $\begin{array}{c}\text { NREL } C_{\mathrm{L}} \\
\text { and } C_{\mathrm{D}}\end{array}$ \\
\hline BEM & -1.13 & -0.41 & -0.52 & -0.24 \\
Coupled model & -1.00 & -0.27 & -0.37 & -0.1 \\
GENUVP & - & -0.25 & - & 0.22 \\
\hline
\end{tabular}

\section{Conclusions}

In this paper, several modifications of a coupled model consisting of a trailed vorticity model for the near-wake and a BEM-based model for the far wake have been presented and validated. Results from the coupled model are compared to free-wake panel code and a BEM model to evaluate the benefits and limitations of the added trailed vorticity modeling.

It has been shown that the acceleration of the model by reducing the number of exponential functions in the trailing wake approximation from two to one is possible with negligible effect on the results. The approach presented here does not change the steady results predicted by the NWM.

An iteration scheme to stabilize the model has been presented. It applies a relaxation factor that is computed dynamically based on the blade discretization and the operating point of the turbine. To evaluate the computed relaxation factors, minimum necessary relaxation factors have been determined by trial and error and the estimated factors are found to be conservative. The iterative process enables stable computations without the need for very small time steps and reduces oscillations of the near-wake induction.

The 2-D shed vorticity modeling, based on thin airfoil theory, has been extended by including the unsteady effects on the bound circulation. Further, it has been found that it is necessary to include airfoil camber in the modeling of the influence of varying inflow velocity on the dynamic angle of attack to obtain good results if the direction of vibration is close to parallel to the inflow direction.

A comparison of pitch step responses of the NREL $5 \mathrm{MW}$ reference turbine using the coupled near- and far-wake model, a BEM model based on the aerodynamics model in HAWC2 and the free-wake panel code GENUVP has been presented. The trailed vorticity modeling in the coupled model gives results closer to the free-wake code than the BEM model during the pitching motion, and for a slow pitching rate a clear improvement is seen in the computation of the overshoot. Fast pitch rates resulted in oscillations due to the motion of the wake in the free-wake code, which could not be achieved in the coupled model due to the prescribed wake assumption. The response to a partial pitch of the outer half of the blade demonstrated the cross-sectional 
aerodynamic coupling, which will have an influence on the load distribution in the presence of trailing edge flaps.

The coupled model agreed better than the BEM model with the free-wake code in all prescribed vibration cases investigated. The main improvement due to the trailed vorticity is found close to the tip of the blade, even in the case of the higher modes investigated. The work response to the edgewise vibrations has been found to be difficult to model if the direction of vibration is close to parallel to the inflow direction. The results in this case compare much better if no drag forces are computed. If drag is included, the coupled model still compares well with the free-wake code close to the blade tip, but there are larger deviations in the results of all models further inboard. In general, the simulations agreed better for out-of-plane vibrations than in-plane vibrations.
The implementation of the coupled near- and far-wake model presented here delivers promising results and will be further investigated and validated against computational fluid dynamics results and measurements in future work. In particular, the more accurate prediction of aerodynamic work for edgewise vibrations is considered to be important for stability analyses and load predictions due to the low aeroelastic damping typically associated with these vibrations.

\section{Data availability}

A repository (Pirrung, 2017) contains the source code of the aerodynamics program used for the BEM and coupled wake model computations. The relevant input data for the NREL 5MW reference turbine are also included. 
Appendix A: Nomenclature

\begin{tabular}{|c|c|c|}
\hline Symbol & Units & Description \\
\hline$A$ & {$[\mathrm{~m}]$} & Amplitude of vibration \\
\hline$A_{1}, A_{2}$ & {$[-]$} & Dynamic inflow weighting factors \\
\hline$A_{\Gamma, 1}, A_{\Gamma, 2}, A_{\Gamma, 3}$ & {$[-]$} & Coefficients in bound circulation step response \\
\hline$A^{*}$ & {$[-]$} & Coefficient of accelerated near-wake model \\
\hline$a_{\mathrm{FW}}$ & {$[-]$} & Far-wake induction factor \\
\hline$a_{\text {ref }}$ & {$[-]$} & Induction factor according to BEM polynomial \\
\hline$b_{\Gamma, 1}, b_{\Gamma, 2}, b_{\Gamma, 3}$ & {$[-]$} & Dimensionless time constants in bound circulation step response \\
\hline$C_{\mathrm{L}}$ & {$[-]$} & Lift coefficient \\
\hline$C_{\mathrm{L}}^{\prime}$ & {$[-]$} & Gradient of lift coefficient with respect to angle of attack \\
\hline$C_{\mathrm{T}}$ & {$[-]$} & Thrust coefficient \\
\hline$c$ & {$[\mathrm{~m}]$} & Chord length \\
\hline$D_{X, s, v}$ & {$\left[\mathrm{~m}^{-} 1\right]$} & Slowly decaying component of induced velocity due to infinitely long vortex arc $v$ with vortex strength 1 at section $s$ \\
\hline$D_{Y, s, v}$ & {$\left[\mathrm{~m}^{-} 1\right]$} & Fast decaying component of induced velocity due to infinitely long vortex arc $v$ with vortex strength 1 at section $s$ \\
\hline $\mathrm{d} w$ & {$\left[\mathrm{~ms}^{-1}\right]$} & Induced velocity due to infinitesimal vortex element \\
\hline$F_{\text {aero }}$ & {$\left[\mathrm{Nm}^{-1}\right]$} & Aerodynamic forces per unit radius \\
\hline$F_{\text {ip }}$ & {$\left[\mathrm{N} \mathrm{m}^{-1}\right]$} & In-plane component of the aerodynamic forces per unit radius \\
\hline$F_{\text {oop }}$ & {$\left[\mathrm{Nm}^{-1}\right]$} & Out-of-plane component of the aerodynamic forces per unit radius \\
\hline$F$ & {$[-]$} & Tip loss factor \\
\hline$f$ & {$[\mathrm{~Hz}]$} & Frequency of vibration \\
\hline$f_{\mathrm{r}}$ & {$[-]$} & Relaxation factor \\
\hline$k$ & {$[-]$} & reduced frequency \\
\hline$k_{\mathrm{FW}}$ & {$[-]$} & Coupling factor \\
\hline$L$ & {$\left[\mathrm{Nm}^{-1}\right]$} & Lift force per unit radius \\
\hline$m$ & {$[\mathrm{~kg}]$} & Modal mass \\
\hline$N_{v}$ & {$[-]$} & Number of vortex arcs trailed from a blade \\
\hline$u_{1}, u_{2}$ & {$\left[\mathrm{~ms}^{-1}\right]$} & Components of time-filtered far-wake-induced velocity \\
\hline$u_{\mathrm{FW}, \mathrm{dyn}}$ & {$\left[\mathrm{ms}^{-1}\right]$} & Time filtered far-wake-induced velocity \\
\hline$u_{\mathrm{FW}, \mathrm{QS}}$ & {$\left[\mathrm{ms}^{-1}\right]$} & Quasi-steady far-wake-induced velocity \\
\hline$u_{\text {tot }}$ & {$\left[\mathrm{ms}^{-1}\right]$} & Total induced velocity due to the combined near and far wake \\
\hline$u_{\infty}$ & {$\left[\mathrm{ms}^{-1}\right]$} & Free wind speed \\
\hline$\Delta t$ & {$[\mathrm{~s}]$} & Time step \\
\hline$v_{\text {ip }}$ & {$\left[\mathrm{ms}^{-1}\right]$} & In-plane component of the blade section velocity \\
\hline$v_{\text {oop }}$ & {$\left[\mathrm{ms}^{-1}\right]$} & Out-of-plane component of the blade section velocity \\
\hline$v_{\mathrm{r}, \text { in-plane }}$ & {$\left[\mathrm{ms}^{-1}\right]$} & In-plane component of relative velocity \\
\hline$v_{\mathrm{r}}$ & {$\left[\mathrm{ms}^{-1}\right]$} & Relative velocity \\
\hline$W_{\text {aero }}$ & {$\left[\mathrm{Nmm}^{-1}\right]$} & Aerodynamic work during one period of oscillation per unit radius \\
\hline$W_{s, v}$ & {$\left[\mathrm{~ms}^{-1}\right]$} & Induced velocity due to vortex $\operatorname{arc} v$ at section $s$ \\
\hline$W_{s}$ & {$\left[\mathrm{~ms}^{-1}\right]$} & Induced velocity due to all vortex arcs at section $s$ \\
\hline$X_{s, v}$ & {$\left[\mathrm{~ms}^{-1}\right]$} & Slowly decaying component of induced velocity due to vortex arc $v$ at section $s$ \\
\hline$x_{1}, x_{2}$ & {$\left[\mathrm{~ms}^{-1}\right]$} & Components of effective angle of attack \\
\hline$x_{\Gamma, 1}, x_{\Gamma, 2}, x_{\Gamma, 3}$ & {$\left[\mathrm{~m}^{2} \mathrm{~s}^{-1}\right]$} & Components of bound circulation \\
\hline$Y_{s, v}$ & {$\left[\mathrm{~ms}^{-1}\right]$} & Fast decaying component of induced velocity due to vortex arc $v$ at section $s$ \\
\hline$\alpha_{\mathrm{QS}}$ & [rad] & Geometric angle of attack \\
\hline$\alpha_{\mathrm{QS}, \text { camber }}$ & [rad] & Geometric angle of attack relative to zero lift angle \\
\hline$\alpha_{0}$ & [rad] & Zero lift angle \\
\hline$\alpha_{\text {eff }}$ & [rad] & Effective angle of attack \\
\hline$\beta$ & [rad] & Angle a blade has rotated since a vortex has been trailed \\
\hline$\Delta \beta$ & [rad] & Angle a blade rotates in one time step \\
\hline$\delta$ & {$[-]$} & Logarithmic decrement \\
\hline$\Gamma_{\mathrm{QS}}$ & {$\left[\mathrm{m}^{2} \mathrm{~s}^{-1}\right]$} & Bound circulation \\
\hline$\Delta \Gamma$ & {$\left[\mathrm{m}^{2} \mathrm{~s}^{-1}\right]$} & Trailed vortex strength \\
\hline$\Omega$ & {$\left[\mathrm{rad} \mathrm{s}^{-1}\right]$} & Rotor speed \\
\hline$\omega$ & {$\left[\mathrm{rad} \mathrm{s}^{-1}\right]$} & Angular velocity \\
\hline$\Phi$ & {$[-]$} & Geometric parameter determining how fast the influence of a trailed vortex element decays \\
\hline$\Phi^{*}$ & {$[-]$} & Geometric parameter of accelerated near-wake model \\
\hline$\tau_{1}, \tau_{2}$ & {$[\mathrm{~s}]$} & Dynamic inflow time constants \\
\hline$\tau$ & {$[-]$} & Dimensionless time in bound circulation step response \\
\hline$\xi$ & {$[-]$} & Damping ratio \\
\hline
\end{tabular}


Competing interests. The authors declare that they have no conflict of interest.

Edited by: G. J. W. van Bussel

Reviewed by: S. Voutsinas and one anonymous referee

\section{References}

Andersen, P. B.: Advanced Load Alleviation for Wind Turbines using Adaptive Trailing Edge Flaps: Sensoring and Control, PhD thesis, The Technical University of Denmark, Roskilde, Denmark, 133 pp., 2010.

Bak, C., Bitsche, R., Yde, A., Kim, T., Hansen, M., Zahle, F., Gaunaa, M., Blasques, J., Døssing, M., Wedel Heinen, J., and Behrens, T.: Light Rotor: The 10-MW reference wind turbine, in: Proceedings of EWEA 2012 - European Wind Energy Conference \& Exhibition, European Wind Energy Association (EWEA), 10 pp., 2012.

Beddoes, T. S.: A near wake dynamic model, Aerodynamics and Aeroacoustics National Specialist Meeting, 25-27 February 1987, Arlington, TX, USA, 1987.

Chassapoyiannis, P. and Voutsinas, S. G.: Aerodynamic Simulations of the flow around a Horizontal Axis Wind Turbine using the GAST software, Deliverable 2.2 of THALIS - NTUA: Development of know-how for the aeroelastic analysis and the design, optimization of wind turbines MIS: 379421 project, 2013.

Dieterich, O., Langer, H., Sneider, O., Imbert, G., Hounjet, M., Riziotis, V., Cafarelli, I., Calvo Alonso, R., Clerc, C., and Pengel, K.: HeliNOVI: Current vibration research activities, 31st European Rotorcraft forum, Florence, Italy, 13-15 September 2005, 83.183.14, 2005.

Hansen, M. H.: Aeroelastic instability problems for wind turbines, Wind Energy, 10, 551-577, doi:10.1002/we.242, 2007.

Hansen, M. H., Gaunaa, M., and Madsen, H. A.: A BeddoesLeishman type dynamic stall model in state-space and indicial formulations, Ris $\varnothing-\mathrm{R}-1354$, Roskilde, Denmark, 2004.

Jonkman, J., Butterfield, S., Musial, W., and Scott, G.: Definition of a 5-MW Reference Wind Turbine for Offshore System Development, National Renewable Energy Laboratory, Tech. rep., NREL/TP-500-38060, 75 pp., 2009.

Kim, T., Hansen, A. M., and Branner, K.: Development of an anisotropic beam finite element for composite wind turbine blades in multibody system, Renew. Energ., 59, 172-183, 2013.

Larsen, T. J. and Hansen, A. M.: How 2 HAWC2, the user's manual, Denmark, Forskningscenter Risoe, Risoe-R-1597, 2007.
Larsen, T. J., Madsen, H. A., Larsen, G. C., and Hansen, K. S.: Validation of the dynamic wake meander model for loads and power production in the Egmond aan Zee wind farm, Wind Energy, 16, 605-624, doi:10.1002/we.1563, 2013.

Madsen, H. A. and Gaunaa, M.: Udvikling af model for 3D induktions- og stallmodellering, Ris $\varnothing-\mathrm{R}-1509$ (DA), Roskilde, Denmark, 2004.

Madsen, H. A. and Rasmussen, F.: A near wake model for trailing vorticity compared with the blade element momentum theory, Wind Energy, 7, 325-341, doi:10.1002/we.131, 2004.

Madsen, H. A., Bak, C., Døssing, M., Mikkelsen, R., and Øye, S.: Validation and modification of the Blade Element Momentum theory based on comparisons with actuator disc simulations, Wind Energy, 13, 373-389, doi:10.1002/we.359, 2010.

Madsen, H. A. (Ed.), Prospathopoulos, J., Voutsinas, S., Riziotis, V., Diakakis, K., Chassapoyiannis, P., Gomez-Iradi, S., Echarri, X. M., Ruiz, A. I., Shen, W. Z., and Sørensen, N. N.: Validation of high rotational speed aerodynamics by wind tunnel tests, Deliverable 2.13, InnWind project, 2015.

Petot, D.: Differential equation modeling of dynamic stall, La Recherche Aerospatiale, English Edn., 59-72, 1989.

Pirrung, G. R.: Aerodynamics code used in Wind Energy Science paper "Comparison of a coupled near- and far-wake model with a free-wake vortex code", Data set, Zenodo, doi:10.5281/zenodo.242498, 2017.

Pirrung, G. R., Hansen, M. H., and Madsen, H. A.: Improvement of a near wake model for trailing vorticity, J. Phys. Conf. Ser., 555, 012083, doi:10.1088/1742-6596/555/1/012083, 2012.

Pirrung, G. R., Madsen, H. A., and Kim, T.: The influence of trailed vorticity on flutter speed estimations, J. Phys. Conf. Ser., 524, 012048, doi:10.1088/1742-6596/524/1/012048, 2014.

Pirrung, G. R., Madsen, H. A., Kim, T., and Heinz, J.: A coupled near and far wake model for wind turbine aerodynamics, Wind Energy, 19, 2053-2069, doi:10.1002/we.1969, 2016.

Riziotis, V. A. and Voutsinas, S. G.: Dynamic Stall on Wind Turbine Rotors: Comparative Evaluation Study of Different Models, in: Proceedings of the 1997 European Wind Energy Conference and Exhibition, Dublin, Ireland, October 1997.

Sørensen, N. N. and Madsen, H. A.: Modelling of transient wind turbine loads during pitch motion, European Wind Energy Association (EWEA), 786-795, 2006.

Voutsinas, S. G.: Vortex Methods in Aeronautics: How to make things work, Int. J. Comput. Fluid D., 20, 3-18, 2006. 